The AstrophysicAl JourNAL, 534:915-933, 2000 May 10

(c) 2000. The American Astronomical Society. All rights reserved. Printed in U.S.A.

\title{
ACCRETIONAL HEATING OF ASYMMETRIC SUPERNOVA CORES
}

\author{
CHRISTOPHER THOMPSON \\ Department of Physics and Astronomy, CB3255, University of North Carolina, Chapel Hill, NC 27599; act@physics.unc.edu \\ Received 1999 May 24; accepted 1999 November 22
}

\begin{abstract}
The role of accretion in heating a stalled bounce shock in a core-collapse supernova is investigated. We show that effective accretional heating causes an asymmetric expansion of the shock, sufficient to impart a net impulse of $\sim 300-400 \mathrm{~km} \mathrm{~s}^{-1}$ to the neutron core. To simplify the analysis, we consider a failed accretion shock. Below such a shock, inward advection is faster than neutrino heating and the usual gain criterion does not suffice to determine a successful explosion. A mechanism that enhances buoyancy and inhibits mixing between hot and cold postshock fluid elements is required to revive the shock. We focus on the response of a magnetic field to the accretion flow. Ram heating and shearing of a low-density, magnetized fluid phase ("M-fluid") is shown to be faster than neutrino cooling. The long duration of the accretion flow compared with the dynamical time allows for a large amplification of the magnetic energy. We calculate the stability of a spherical shock in the presence of a low-density hydrostatic atmosphere below it and show that below a critical atmospheric density the shock is unstable to a global Rayleigh-Taylor mode. We then calculate the equilibrium structure of this Rayleigh-Taylor plume as it accumulates energy and the critical size beyond which quasi-static expansion is no longer possible and its outer boundary converts to a running shock. Accretion continues while the shock expands, and an energy of $\sim 10^{51}$ ergs is a direct consequence of the efficiency of ram heating close to the neutron core. The linear momentum imparted to the core is directly related to the mass profile of the precollapse core and explains the proper motions of (most) radio pulsars. We also estimate the net circulation imparted to the last $0.1-0.2 M_{\odot}$ of collapsing material, which appears sufficient to torque the core down to a spin period of $1-100 \mathrm{~ms}$. The effect of photodissociation on the shock jump conditions is calculated, and the implications for nucleosynthesis of iron peak elements are considered. Finally, the residual magnetic field advected out into the eventual supernova remnant is compared with the field generated by a rapidly spinning neutron star.
\end{abstract}

Subject headings: accretion, accretion disks — shock waves — stars: magnetic fields stars: neutron - supernovae: general

\section{INTRODUCTION}

The mechanism that powers the explosion of a massive star - a supernova of Type II or Ibc - has not yet been fully captured. The release of gravitational binding energy through the formation of a neutron star is ultimately responsible (Baade \& Zwicky 1934). Several suggestions have been made over the years as to how $\sim 0.3 \%$ of this energy is converted into kinetic energy, and it is clear that more than one of these may, at some level, be important. The collapse of the star's core upon the depletion of nuclear fuel creates a bounce shock that propagates outward (Colgate \& Johnson 1960), but the shock appears to lose energy to nuclear dissociation and neutrino cooling before it can blow off the outermost layers of the star (e.g., Mazurek, Cooperstein, \& Kahana 1982). A stalled shock could be revived by neutrino radiation from the cooling neutron core, which heats the material directly behind the shock (Colgate \& White 1966; Bethe \& Wilson 1985). Various authors have speculated about rotation: for detailed calculations, see especially the pioneering work of Leblanc \& Wilson (1970), as well as Janka \& Monchmeyer (1989), MacFadyen \& Woosley (1999), and Khokhlov et al. (1999). The rotational energy must, however, be fine-tuned: if the neutron star is born spinning more rapidly than $P \sim 5$ $\mathrm{ms}$, it will overenergize the surrounding nebula (Duncan \& Thompson 1992, hereafter DT92).

Neutrino heating of a thin mass shell just interior to the stalled shock induces a successful explosion in some numerical simulations (Bethe \& Wilson 1985; Wilson \&
Mayle 1993; Burrows, Hayes, \& Fryxell 1995, hereafter BHF; Janka \& Müller 1996, hereafter JM96) but not in others (Mezzacappa et al. 1998, hereafter M98). The success or failure of the shock appears to depend sensitively on $L_{v_{e}, \bar{v}_{e}}$, on the radius at which the shock stalls (which, in turn, depends on the mass of the precollapse core and the equation of state above nuclear density), and on the effects of general relativity. Although successful explosions have been obtained for relatively low-mass cores and high $L_{v_{e}, \bar{v}_{e}}$, some models with high $L_{v_{e}, \bar{v}_{e}}$ result in failures (M98). The efficacy of neutrino heating may be enhanced by convection (Epstein 1979), both inside the neutrinosphere (by increasing the energy flux and temperature of the emerging neutrinos: Burrows 1987; Wilson \& Mayle 1993; Keil, Janka, \& Müller 1996, hereafter KJM) and in between the neutrinosphere and the shock (Herant, Benz, \& Colgate 1992; Miller, Wilson, \& Mayle 1993; BHF; JM96). A cogent argument linking the success of the shock to convection, however, has not yet been given.

The incompleteness in our understanding of corecollapse supernovae is highlighted by two well-known observational facts: many young neutron stars are rapidly rotating $\left(P_{\mathrm{ns}} \sim 0.01-0.1 \mathrm{~s}\right)$ and rapidly translating $\left(V \sim 250-450 \mathrm{~km} \mathrm{~s}^{-1}\right.$; Lyne \& Lorimer 1994; Hansen \& Phinney 1997; Cordes \& Chernoff 1998; Fryer, Burrows, \& Benz 1998). The associated kinetic energies are $E_{\text {rot }} \sim$ $10^{50}\left(P_{\mathrm{ns}, \text { initial }} / 10 \mathrm{~ms}\right)^{-2}$ ergs and $10^{48}\left(V / 300 \mathrm{~km} \mathrm{~s}^{-1}\right)^{2}$ ergs, respectively. The rotation and kicks have been variously ascribed to large-scale convective motions, or asymmetries 
in neutrino radiation, but only as a secondary consequence of the supernova mechanism. In scenarios where rotation actually drives the explosion, a convincing explanation for rapid rotation in the precollapse core has yet to be given. It would be more appealing to find a supernova mechanism that leads directly to large kicks.

The sensitivity to parameters in the numerical simulations combined with the absence of a convincing analytical prescription for the breakout of the supernova shock, and the absence of a convincing mechanism for neutron star kicks, leads us to consider whether some key physics has not yet been incorporated into numerical simulations. It was noted several years ago that a very strong, small-scale magnetic field $\left(B \sim 10^{15} \mathrm{G}\right)$ is probably present in the vicinity of the neutrinosphere (Thompson \& Duncan 1993, hereafter TD93). Reconnected magnetic fields provide an efficient source of pressure support because the energy deposited in the field is not readily converted back to neutrinos (C. Thompson \& N. W. Murray 2000, in preparation). The origin of this magnetic field was ascribed to a violent and persistent convective instability inside the neutrinosphere. Both mixing length arguments (Burrows 1987; TD93) and explicit radiation-hydrodynamical simulations (KJM) suggest that the convective velocities approach 1-3 $\times 10^{8} \mathrm{~cm} \mathrm{~s}^{-1}$. However, reconnection must release a much larger fraction of the bolometric output of the supernova core $\left(\varepsilon_{\mathrm{mag}} \sim 0.003-0.01\right)$ than it appears to accomplish in the nonthermal atmosphere of the Sun (the chromosphere of which radiates only $\sim 10^{-4}$ of the bolometric output).

In this paper, we go on to identify a second strong instability, involving rapid accretion onto a newly formed neutron core, which pumps energy into a buoyant, magnetized fluid in the vicinity of a stalled accretion shock. This amplification mechanism relies on an almost unique property of a supernova core: the rapid, quasi-spherical accretion that deposits a dense atmosphere outside the neutrinosphere during the first stages of the collapse. In this picture, the magnetic field has two key roles: it maintains the buoyancy of a component of the flow and, at the same time, it prevents complete mixing of hot and cold fluid below the shock. Given a fallback rate of $\sim 0.3 M_{\odot} \mathrm{s}^{-1}$, a total energy input to the shock of $\sim 10^{51}$ ergs in the first few tenths of a second after bounce appears plausible. An attractive feature of this heating mechanism is that the buoyancy force acting on the magnetized fluid (the " $M$ fluid") is greatly reduced when the shock succeeds, accretion is followed by a neutrino-driven wind, and the hydrostatic pressure outside the neutrinosphere drops below the magnetic pressure. This feedback suggests that enough energy is injected to blow off the outer layers of the star, but not too much more.

To simplify the calculations, we focus on the case where the neutrinos have failed to revive the bounce shock. The fallback rate increases rapidly with the mass of the progenitor star, suggesting that neutrino heating is ineffective above some critical mass (Woosley \& Weaver 1995, hereafter WW95). We present some simple analytical arguments explaining why neutrino heating will not revive the shock after a quasi-steady accretion flow onto the neutron core is established. The derived accretion flow is consistent with asymptotic behavior seen in the simulations of M98, after the shock begins to fall back toward the neutron core. A "gain region" (where neutrino heating overcomes cooling;
Bethe 1990) persists below the shock; but the timescale for neutrino heating is longer than the time for material to flow inward. In this situation, neutrino heating is too slow to impede the subsonic accretion flow below the shock, as long as the gain region is well mixed. We go on to show that a buildup of entropy and magnetic pressure below the shock triggers a large-scale Rayleigh-Taylor mode that both energizes the supernova shock and imparts asymmetries near the rarefaction wave that are directly responsible for both pulsar rotation and kicks. Very rapid initial rotation of the neutron star $\left(P_{\mathrm{ns}} \sim 1-100 \mathrm{~ms}\right)$ becomes a plausible aftereffect of the collapse, giving further impetus to models that associate very strong magnetism $\left(B>10^{14} \mathrm{G}\right)$ with rapid initial rotation (DT92) and require a birthrate of $\sim 1$ $3 \times 10^{-3}$ per year of such objects (Kulkarni et al. 1994; Kouveliotou et al. 1998; Thompson et al. 1999).

These considerations may also be applicable to supernova cores in which neutrino heating is an important source of energy for the shock. It is possible that, in some circumstances, accretional heating and neutrino heating are competitive. Analytical treatments of the buildup of energy below a successful, neutrino-heated shock (e.g., Bethe 1997) generally assume a long residency time. The corresponding numerical simulations appear to justify this assumption: neutrino heating revives the shock before a steady accretion flow onto the neutron core is established. Nonetheless, a more precise understanding of the limiting effect of inward advection on neutrino heating is needed to define the initial conditions that produce a successful neutrino-heated shock.

Any neutron core that acquires a centrifugally supported torus will release considerably more rotational energy than does a hydrostatically supported star of equal angular momentum. For that reason, an energetic jet is a plausible consequence of any asymmetry that leads to the formation of a torus; rapid rotation of the pre-supernova core is not an obvious prerequisite. This raises the possibility that some supernovae that give birth to radio pulsars emit energetic jets during the first minutes of the explosion. Nonetheless, a (smaller) fraction of supernova cores can be expected to rotate rapidly before they collapse. For example, a Type $\mathrm{Ib} / \mathrm{c}$ supernova the progenitor of which corotates with a close binary companion is guaranteed to retain the necessary angular momentum (Thompson 1994; Nakamura 1998). Directed energy output from a rapidly rotating supernova core has been simulated recently by MacFadyen \& Woosley (1999) and Khokhlov et al. (1999), with a focus on the possible connection with some (intrinsically faint) $\gamma$-ray burst sources.

The plan of this paper is as follows. In $\S 2$ we calculate the properties of the accretion flow below a failed shock, including the effects of photodissociation, neutrino heating and cooling, and convection. Section 3 treats the response of a low-density M-fluid to the combined effects of the accretion ram and heating by core neutrinos and considers the possibility of a convective dynamo outside the neutrinosphere. In $\S 4$, we argue that the accumulation of energy in the M-fluid results in an asymmetric and quasi-static Rayleigh-Taylor expansion of the shock through the accretion flow. We quantify the conditions necessary for such an expansion and the equilibrium shape of the shock. A shock energy of $\sim 10^{51}$ ergs follows directly from the heating rate near the neutron core. The implications of this asymmetric expansion for pulsar proper motions and rotation are analyzed in $\S 5$. Two further observational tests are presented in $\S 6$ : the 
influence of the shock heating mechanism on explosive nucleosynthesis of iron peak elements and the strength of the magnetic field advected out in the supernova ejecta.

\section{NEUTRINO HEATING BELOW A STALLED SHOCK}

We first consider the structure of the flow surrounding a shock that has not been revived by neutrinos and has collapsed to $R_{\mathrm{sh}} \sim 100 \mathrm{~km}$. The range of pre-supernova core masses and compositions that lead to successful neutrino heating is not presently known, so we will simply parameterize the density profile of the core. We find that a reduction in the shock radius (caused, e.g., by the effects of relativistic gravity; De Nisco, Bruenn, \& Mezzacappa 1998) shortens the neutrino cooling time behind the shock not only in absolute value, but also relative to the flow time and to the time for heating by core neutrinos. This suggests that a smaller shock leads to weaker neutrino heating.

\subsection{A Shock Stalled at Small Radius}

Consider a stationary shock at radius $R_{\mathrm{sh}}$ in a spherical accretion flow. The accretion rate $\dot{M}=4 \pi R^{2} \rho V$ declines slowly on the dynamical time $\left(R_{\mathrm{sh}}^{3} / 2 G M_{\text {core }}\right)^{1 / 2}$. Most of the pressure in the postshock flow is provided by radiation and $e^{ \pm}$pairs when $R_{\mathrm{sh}}$ sits inside $\sim 100 \mathrm{~km}$ and $\dot{M}$ lies below $\sim 1 M_{\odot} \mathrm{s}^{-1}$. Furthermore, photodissocation into neutrons and protons behind the shock ${ }^{1}$ is immediate and complete since the thermal energy absorbed per infalling nucleon ( 8.8 $\mathrm{MeV}$ for ${ }^{56} \mathrm{Fe}$, and $8.0 \mathrm{MeV}$ for ${ }^{16} \mathrm{O}$ ) is a fraction of the gravitational binding energy

$$
\frac{G M m_{n}}{R_{\mathrm{sh}}}=19\left(\frac{R_{\mathrm{sh}}}{100 \mathrm{~km}}\right)^{-1} \mathrm{MeV} \text { baryon }^{-1}
$$

at this small a radius. The jump conditions across the accretion shock are then

$$
\frac{11}{12} a T_{2}^{4}+\frac{\rho_{2} T_{2}}{m_{n}}+\rho_{2} V_{2}^{2}=\rho_{1} V_{1}^{2}
$$

and

$$
V_{2}\left(\frac{11}{3} a T_{2}^{4}+\frac{5}{2} \frac{\rho_{2} T_{2}}{m_{n}}+\frac{1}{2} \rho_{2} V_{2}^{2}\right)=\frac{1}{2} \rho_{1} V_{1}^{3}-\varepsilon \rho_{1} V_{1} .
$$

Here 1 labels the flow just upstream of the shock, 2 just below the shock, and $\varepsilon$ is the energy absorbed by photodissociation (per unit mass). The equation of mass conservation is $\rho_{2} V_{2}=\rho_{1} V_{1}$, as usual.

Because the energy density in radiation and pairs is a strong function of $T_{2}$, we may treat the nucleon pressure postshock as a perturbation to the total pressure. We define the effective dissociation energy

$$
\varepsilon^{\prime}=\varepsilon-\frac{3}{2} \frac{T_{2}^{*}}{m_{n}}
$$

where $T_{2}^{*}$ is the postshock temperature calculated neglecting the nucleon pressure. The negative sign on the right side of equation (4) arises because photodissociation and the nucleon pressure have opposing effects on the shock compression. Photodissociation increases the compression by removing thermal energy from the flow; whereas the compression decreases as nonrelativistic particles (nucleons) contribute a larger fraction of the postshock pressure. The shock.

${ }^{1} \mathrm{We}$ assume that the electron fraction $Y_{e}$ is conserved through the compression ratio across the shock is then

$$
\mathscr{R} \equiv \frac{\rho_{2}}{\rho_{1}} \simeq \frac{7}{4-3\left[1+\left(14 \varepsilon^{\prime} / 9 V_{1}^{2}\right)\right]^{1 / 2}} .
$$

The postshock enthalpy is dominated by relativistic particles until $\varepsilon^{\prime}$ approaches $\frac{1}{2} V_{1}^{2}$. Except in that limit, the postshock temperature is fixed by the upstream ram and is not much altered by photodissociation:

$$
\begin{aligned}
\left(P_{e^{ \pm}}+P_{\gamma}\right)_{2}= & \frac{11}{12} a T_{2}^{4} \quad\left(T_{2} \gg m_{e} c^{2}\right) \\
\simeq & \frac{3}{7}\left\{1+\left[1+\left(\frac{14 \varepsilon^{\prime}}{9 V_{1}^{2}}\right)\right]^{1 / 2}\right\} \rho_{1} V_{1}^{2} .
\end{aligned}
$$

Close to the core, the accretion flow will have accelerated close to the free-fall speed (Yahil \& Lattimer 1982), so that

$$
\left[\rho_{1} V_{1}^{2}\right]\left(R_{\mathrm{sh}}\right) \simeq \frac{\dot{M}}{4 \pi}\left(\frac{2 G M_{\text {core }}}{R_{\mathrm{sh}}^{5}}\right)^{1 / 2} .
$$

The numerical values become

$$
\begin{aligned}
\rho_{2}= & 2.6 \times 10^{9}\left(\frac{\mathscr{R}}{10}\right)\left(\frac{\dot{M}}{M_{\odot} \mathrm{s}^{-1}}\right)\left(\frac{R_{\mathrm{sh}}}{100 \mathrm{~km}}\right)^{-3 / 2} \\
& \times\left(\frac{M_{\text {core }}}{1.4 M_{\odot}}\right)^{-1 / 2} \mathrm{~g} \mathrm{~cm}^{-3}
\end{aligned}
$$

and (in the limit $\varepsilon^{\prime} \rightarrow 0$ )

$$
\begin{aligned}
T_{2}^{*}= & 2.9\left(\frac{\dot{M}}{M_{\odot} \mathrm{s}^{-1}}\right)^{1 / 4}\left(\frac{R_{\mathrm{sh}}}{100 \mathrm{~km}}\right)^{-5 / 8} \\
& \times\left(\frac{M_{\text {core }}}{1.4 M_{\odot}}\right)^{1 / 8} \mathrm{MeV} .
\end{aligned}
$$

Our assumption that the electrons behind the shock are nondegenerate can be checked by evaluating the electron chemical potential,

$$
\begin{aligned}
\left(\frac{\mu_{e}}{T}\right)_{2} \simeq & \frac{3 Y_{e} \rho_{2} / m_{n}}{\left(T_{2}^{*}\right)^{3}}=0.8\left(\frac{Y_{e}}{0.5}\right)\left(\frac{\mathscr{R}}{10}\right) \\
& \times\left(\frac{\dot{M}}{M_{\odot} \mathrm{s}^{-1}}\right)^{1 / 4}\left(\frac{R_{\mathrm{sh}}}{100 \mathrm{~km}}\right)^{3 / 8}\left(\frac{M_{\text {core }}}{1.4 M_{\odot}}\right)^{-7 / 8} .
\end{aligned}
$$

Recall that the approximate boundary between the degenerate and nondegenerate regimes is $\mu_{e} / T \sim \pi$. To check our assumption that pairs and photons dominate the pressure, note that

$$
\begin{aligned}
\frac{\left(P_{e^{ \pm}}+P_{\gamma}\right)_{2}}{\rho_{2} T_{2} / m_{n}} & =\frac{11 \pi^{2}}{60}\left(\frac{Y_{e}}{\mu_{e} / T}\right)_{2} \\
& \simeq \frac{6}{35}\left(\frac{\mathscr{R}}{10}\right)^{-1} \frac{G M_{\text {core }} m_{n}}{R_{\mathrm{sh}} T_{2}},
\end{aligned}
$$

or, on substituting equation (9),

$$
\begin{aligned}
\frac{\left(P_{e^{ \pm}}+P_{\gamma}\right)_{2}}{\rho_{2} T_{2} / m_{n}}= & 1.2\left(\frac{\dot{M}}{M_{\odot} \mathrm{s}^{-1}}\right)^{-1 / 4}\left(\frac{R_{\mathrm{sh}}}{100 \mathrm{~km}}\right)^{-3 / 8} \\
& \times\left(\frac{\mathscr{R}}{10}\right)^{-1}\left(\frac{M_{\text {core }}}{1.4 M_{\odot}}\right)^{7 / 8}
\end{aligned}
$$




\subsection{Competition between Neutrino Heating and Inward Advection}

Absorption of electron neutrinos by free neutrons deposits energy outside the neutrinosphere ( $v$-sphere) at the rate

$$
\begin{aligned}
\dot{E}_{v_{e}+n \rightarrow p+e^{-}}= & \frac{390}{f}\left(\frac{L_{v_{e} 52}}{4}\right)\left(\frac{\left\langle\varepsilon_{v_{e}}^{2}\right\rangle}{200 \mathrm{MeV}^{2}}\right) \\
& \times\left(\frac{R}{100 \mathrm{~km}}\right)^{-2} \mathrm{MeV} \text { neutron }^{-1} \mathrm{~s}^{-1}
\end{aligned}
$$

(Bethe \& Wilson 1985). ${ }^{2}$ The dependence on the mean square electron neutrino energy $\left\langle\varepsilon_{v_{e}}^{2}\right\rangle$ originates in the weak interaction cross section. The expression for proton heating $\left(\bar{v}_{e}+p \rightarrow n+e^{+}\right)$is obtained by replacing $L_{v_{e}}$ and $\left\langle\varepsilon_{v_{e}}^{2}\right\rangle$ with $L_{\bar{v}_{e}}$ and $\left\langle\varepsilon_{\bar{v}_{e}}^{2}\right\rangle$. Here $f \simeq \frac{1}{4}$ for isotropic emission at the $v$-sphere, increasing to $f=1$ farther out where the neutrinos free stream. The net heating rate caused by absorption of $v_{e} / \bar{v}_{e}$ on free neutrons ( $Y_{n}$ per nucleon) and protons ( $Y_{p}$ per nucleon) is

$$
\dot{Q}^{v N}=\left(\dot{E}_{v_{e}+n \rightarrow p+e^{-}} Y_{n}+\dot{E}_{\bar{v}_{e}+p \rightarrow n+e^{+}} Y_{p}\right) \frac{\rho}{m_{n}}
$$

per unit volume.

Heating of free nucleons is compensated by cooling caused by the inverse process of electron (positron) capture, $e^{-}+p \rightarrow v_{e}+n\left(e^{+}+n \rightarrow \bar{v}_{e}+p\right)$

$$
\begin{aligned}
\dot{Q}^{e N} & \equiv \dot{Q}_{e^{-}+p \rightarrow v_{e}+n}+\dot{Q}_{e^{++n \rightarrow \bar{v}_{e}+p}} \\
& =1.4 \times 10^{36} \rho_{12} T_{\mathrm{MeV}}^{6}\left(Y_{p}+Y_{n}\right) \mathrm{MeV} \mathrm{cm}^{-3} \mathrm{~s}^{-1},
\end{aligned}
$$

where $T=T_{\mathrm{MeV}} \mathrm{MeV}$. The equilibrium temperature at which heating balances cooling is

$$
T_{\text {eq }} \simeq 2.35 f^{-1 / 6}\left[\frac{\mathscr{L}_{52}}{4\left(Y_{n}+Y_{p}\right)}\right]^{1 / 6}\left(\frac{R}{100 \mathrm{~km}}\right)^{-1 / 3} \mathrm{MeV} \text {. }
$$

We define the convenient dimensionless parameter

$$
\begin{aligned}
\mathscr{L}_{52} & \equiv Y_{n} L_{v_{e} 52}\left[\frac{\left\langle\varepsilon_{v_{e}}^{2}\right\rangle}{200(\mathrm{MeV})^{2}}\right] \\
& +Y_{p} L_{\bar{v}_{e}} 52\left[\frac{\left\langle\varepsilon_{\bar{v}_{e}}^{2}\right\rangle}{200(\mathrm{MeV})^{2}}\right] .
\end{aligned}
$$

When matter accretes at a rate as large as $\dot{M} \sim 10^{-1}-1$ $M_{\odot} \mathrm{s}^{-1}$, postshock neutrino cooling is dominated by pair capture on nucleons (eq. [15]) rather than by neutrino pair emission $\left(e^{+}+e^{-} \rightarrow v+\bar{v}\right)$, for which

$$
\dot{Q}^{e^{ \pm}}=8 \times 10^{30} T_{\mathrm{MeV}}^{9} \mathrm{MeV} \mathrm{cm} \mathrm{cm}^{-3} \mathrm{~s}^{-1}
$$

near $T \sim 3 \mathrm{MeV}$ (Schinder et al. 1987). In general,

$$
\frac{\dot{Q}^{e^{ \pm}}}{\dot{Q}^{e N}}=0.045 \frac{P_{e^{ \pm}}+P_{\gamma}}{\rho T / m_{n}}
$$

in a medium with nondegenerate electrons. Just behind the shock,

$$
\begin{aligned}
\frac{\left(\dot{Q}^{e N}\right)_{2}}{\left(\dot{Q}^{e^{ \pm}}\right)_{2}}= & 13\left(\frac{\dot{M}}{M_{\odot} \mathrm{s}^{-1}}\right)^{1 / 4}\left(\frac{R_{\mathrm{sh}}}{100 \mathrm{~km}}\right)^{3 / 8} \\
& \times\left(\frac{\mathscr{R}}{10}\right)\left(\frac{M_{\text {core }}}{1.4 M_{\odot}}\right)^{-7 / 8} .
\end{aligned}
$$

\footnotetext{
${ }^{2}$ We focus in this paper on the part of the supernova that is optically thin to neutrinos and are not concerned with how the position of the $v$-sphere varies with neutrino type.
}

This contrasts with the neutrino-cooled accretion models of Houck \& Chevalier (1991) and Brown (1995), in which $\dot{M} \lesssim 10^{-4} M_{\odot} \mathrm{s}^{-1}$ and cooling is dominated by pair emission.

The importance of heating by the core neutrinos, relative to direct cooling of the postshock material (by $e^{ \pm}$capture), can be determined by comparing the postshock temperature (eq. [9]) with the equilibrium temperature (eq. [16]):

$$
\begin{aligned}
\frac{T_{2}^{*}}{T_{\text {eq }}}= & 1.3 f^{1 / 6}\left(\frac{\dot{M}}{M_{\odot} \mathrm{s}^{-1}}\right)^{1 / 4} \\
& \times\left(\frac{R_{\mathrm{sh}}}{100 \mathrm{~km}}\right)^{-7 / 24}\left(\frac{\mathscr{L}_{52}}{4}\right)^{-1 / 6} .
\end{aligned}
$$

Heating dominates cooling where $T_{2}^{*}<T_{\mathrm{eq}}$. Equation (21) is consistent with the $15 M_{\odot}$ collapse model of M98, in which the shock failed but a narrow gain region was nonetheless present below it. Substituting the appropriate parameters $\dot{M} \sim 0.3 M_{\odot}, L_{v_{e} 52}=4$, and $R_{\mathrm{sh}}=120 \mathrm{~km}$ for a postcollapse time $t=0.3 \mathrm{~s}$, we deduce $T_{2}^{*} / T_{\mathrm{eq}} \simeq$ $0.9 f^{1 / 6}<1$. More generally, equation $(21)$ has the interesting implication that cooling overcomes heating at higher accretion rates (as expected in the cores of more massive progenitor stars).

This dominance of neutrino heating over cooling has been argued by Bethe \& Wilson (1985) and Bethe (1997) to cause an energetic explosion. However, a convincing analytic prescription for the success of the shock has yet to be given. The "gain" criterion, as applied to core-collapse simulations, generally presupposes that the material accreted through the shock remains in the gain region long enough to be heated substantially by the core neutrinos. The numerical simulations of Wilson \& Mayle (1993) and JM96 find both a successful shock and a substantial dwelling time of the accreted material. Some recent simulations of M98 find neither. The following considerations do not directly address the critical, transient phase in core-collapse simulations where the failure or success of the shock is determined. However, they agree well with the asymptotic behavior of the postshock flow in the simulations of M98 and so can be applied to a situation where neutrino heating has failed to revive the shock. They also highlight the fact that strong neutrino-driven convection continues even if the bounce shock should turn into an accretion shock.

Let us compare the time for core neutrinos to increase the pressure of the shocked material,

$$
\left(\tau_{\text {heat }}^{v N}\right)_{2}=\frac{(11 / 4) a T_{2}^{4}}{\left(\dot{Q}^{v N}\right)_{2}},
$$

with the time for the same material to flow across one pressure scale height $\ell_{P} \simeq \frac{1}{4} R_{\mathrm{sh}}$,

$$
\left(\tau_{\text {flow }}\right)_{2}=\frac{(1 / 4) R_{\mathrm{sh}}}{V_{2}}=\frac{\mathscr{R}}{4}\left(\frac{R_{\mathrm{sh}}^{3}}{2 G M_{\text {core }}}\right)^{1 / 2} .
$$

One finds that the heating time is substantially longer,

$$
\begin{aligned}
\frac{\left(\tau_{\text {heat }}^{v N}\right)_{2}}{\left(\tau_{\text {flow }}\right)_{2}}= & 6.3\left(\frac{\mathscr{R}}{10}\right)^{-2}\left(\frac{R_{\text {sh }}}{100 \mathrm{~km}}\right)^{1 / 2} \\
& \times\left(\frac{\mathscr{L}_{52}}{4}\right)^{-1}\left(\frac{M_{\text {core }}}{1.4 M_{\odot}}\right)^{3 / 2} .
\end{aligned}
$$


Heating by the core neutrinos is not fast enough to stall the accretion flow. Convective stresses below the shock are likewise smaller than the thermal pressure $(\S 2.3)$. It will also turn out to be important that direct cooling by the accreted material is slow immediately below the shock:

$$
\begin{aligned}
\frac{\left(\tau_{\text {cool }}^{e N}\right)_{2}}{\left(\tau_{\text {flow }}\right)_{2}}= & \frac{(11 / 4) a T_{2}^{4}}{\left(\dot{Q}^{e N} \tau_{\text {flow }}\right)_{2}} \\
= & 1.9\left(\frac{\mathscr{R}}{10}\right)^{-2}\left(\frac{\dot{M}}{M_{\odot} \mathrm{s}^{-1}}\right)^{-3 / 2} \\
& \times\left(\frac{R_{\text {sh }}}{100 \mathrm{~km}}\right)^{5 / 4}\left(\frac{M_{\text {core }}}{1.4 M_{\odot}}\right)^{3 / 4} .
\end{aligned}
$$

As the accreted material flows downward past the gain region, both neutrino heating and cooling rates increase. The layer immediately below the shock is very nearly hydrostatic and adiabatic, $\rho \simeq \rho_{2}\left(T / T_{2}\right)^{3}, P \simeq P_{2}\left(T / T_{2}\right)^{4}$. The Euler equation can be approximated by

$$
-\frac{G M_{\text {core }}}{R^{2}} \simeq \frac{1}{\rho} \frac{d P}{d R} \simeq \frac{48}{7 \mathscr{R}}\left(\frac{G M_{\text {core }}}{R_{\text {sh }} T_{2}}\right) \frac{d T}{d R}
$$

which integrates to

$$
\frac{T}{T_{2}} \simeq\left(\frac{R_{\text {sh }}}{R}\right)
$$

when $\mathscr{R} \simeq 7$. The relation in equation (27) breaks down when the postshock fluid contains two phases, one of which has a low density and dominates the volume (§4.4).

Further inside the shock, the cooling time decreases as $\tau_{\text {cool }}^{e N} \propto T^{-2} \rho^{-1} \propto R^{5}$. By contrast, the heating time decreases only as $\tau_{\text {heat }}^{v N} \propto R^{2}$ and the dynamical time remains approximately constant, $R / V \propto \rho R^{\mathbf{3}} / \dot{M} \sim R^{0}$. The base of this adiabatic flow (radius $R_{\text {coor }}$ ) is identified with the outer boundary of the neutron star's atmosphere, within which the electrons become degenerate and rapid electron captures induce a sharp positive gradient $d Y_{e} / d R$ (Wilson \& Mayle 1993; JM96; M98). Thus, the equilibrium standoff distance of the shock from the center of the neutron core is given by $\left(\boldsymbol{R}_{\mathrm{sh}} / \boldsymbol{R}_{\text {cool }}\right)^{5} \simeq \tau_{\text {cool }}^{e N}\left(\boldsymbol{R}_{\mathrm{sh}}\right) / \tau_{\text {flow }}\left(\boldsymbol{R}_{\mathrm{sh}}\right)$, or

$$
\begin{aligned}
\frac{R_{\mathrm{sh}}}{R_{\text {cool }}} & =2.4\left(\frac{\dot{M}}{0.1 M_{\odot} \mathrm{s}^{-1}}\right)^{-2 / 5}\left(\frac{R_{\mathrm{cool}}}{50 \mathrm{~km}}\right)^{1 / 3} \\
& \times\left(\frac{\mathscr{R}}{10}\right)^{-8 / 15}\left(\frac{M_{\text {core }}}{1.4 M_{\odot}}\right)^{1 / 5} .
\end{aligned}
$$

\subsection{Convection Outside the Neutrinosphere}

Material that passes through a failed shock flows inward faster than it can be heated by the core neutrinos or cooled by $e^{ \pm}$capture (eqs. [24] and [25]). As a result, the flow is nearly adiabatic in the absence of neutrino heating and is susceptible to a convective instability driven by a limited amount of heating outside the gain radius. ${ }^{3}$ Thus, the role of neutrino heating in smoothing out the entropy profile has perhaps been overemphasized. Nonetheless, convection

\footnotetext{
${ }^{3}$ At large $\dot{M}$ and small $R_{\text {sh }}$, neutrino heating is slower than cooling just below the shock, and a gain radius cannot be defined (eq. [16]).
}

does reduce the magnitude of the entropy gradient below the level that would be produced by direct neutrino cooling acting alone on the accretion flow.

The convective velocity $V_{\text {con }}$ below the shock can be estimated by equating the kinetic energy $\frac{1}{2} \rho_{2} V_{\text {con }}^{2}$ with the thermal energy injected by core neutrinos within the rise time $\ell_{P} / V_{\text {con }}=R / 4 V_{\text {con }}$,

$$
\frac{1}{2} \rho_{2} V_{\text {con }}^{2} \sim \frac{\dot{Q}^{v N} R}{4 V_{\text {con }}} .
$$

The pressure $P_{2} \simeq \rho_{1} V_{1}^{2}$ and sound speed $c_{s 2}^{2} \simeq 4 P_{2} / 3 \rho_{2}$ below the shock can be used to calculate the Mach number of the mean accretion flow,

$$
(M a)_{2}=\frac{V_{2}}{c_{s 2}} \simeq \sqrt{\frac{3}{4 \mathscr{R}}} \sim 0.3 .
$$

Substituting $\dot{Q}^{v N}=3 P_{2} / \tau_{\text {heat }}^{v N}$ in equations (29) and (30) yields the convective Mach number

$$
(M a)_{\text {con }}=\frac{V_{\text {con }}}{c_{s 2}} \simeq \frac{3^{5 / 6}}{2^{2 / 3}} \mathscr{R}^{-1 / 6}\left(\frac{\tau_{\text {flow }}}{\tau_{\text {heat }}^{v N}}\right)^{1 / 3} \sim 0.6 .
$$

The ratio of convective to hydrostatic stresses is

$$
\begin{aligned}
\frac{\rho_{2} V_{\text {con }}^{2}}{P_{2}}= & \frac{4}{3}(M a)_{\text {con }}^{2} \simeq 0.4\left(\frac{\mathscr{R}}{10}\right)\left(\frac{R_{\mathrm{sh}}}{100 \mathrm{~km}}\right)^{-1 / 3} \\
& \times\left(\frac{\mathscr{L}_{52}}{4}\right)^{2 / 3}\left(\frac{M_{\text {core }}}{1.4 M_{\odot}}\right) .
\end{aligned}
$$

As the shock expands, convective stresses contribute less to the pressure support below the shock.

\section{ACCRETION-DRIVEN MAGNETIC HEATING}

Effective heating of the layer below the accretion shock requires (1) a mechanism to maintain the buoyancy of heated material and (2) a mechanism to prevent convective mixing of low-density, high-entropy material with the accretion flow. These two requirements are, of course, closely related. Our central argument is that both requirements can be satisfied by allowing a portion of the postshock fluid to be strongly magnetized. The combined effect of the accretion ram and absorption of core neutrinos is to heat this low-density component while simultaneously stretching the magnetic field. This section begins with a brief discussion of the response of a magnetic field to the convective motions in the supernova core, including some new comments on the role of convection outside the $v$-sphere. We then show that direct neutrino cooling is too slow to compensate ram heating except at very high $\dot{M}$ and that ram heating is typically a few times stronger than direct neutrino heating. This heating mechanism is, in some sense, the inverse of the heat engine envisaged by Herant et al. (1992). The heavy, infalling material passes through the accretion shock without gaining enough energy from neutrinos to be expelled; but in the process, its gravitational binding energy is (partly) transferred to a second, higher entropy component that drives the shock.

\subsection{Amplification of Magnetic Fields by Convection}

The supernova core is subject to a violent convective instability below the shock, energized by the free-streaming 
core neutrinos (Bethe 1990; Herant et al. 1992; Miller et al. 1993; JM96; M98), and a distinct instability inside the $v$-sphere, driven by diffusive neutrino cooling (Burrows 1987; TD93; KJM). We briefly review the response of a magnetic field to these convective motions.

Even though the rotation of the gain region is likely to be slow, the convection motions there can amplify a magnetic field to enormous strengths. The argument is based on a direct phenomenological scaling rather than purely theoretical considerations and directly parallels that given in TD93. One observes in the convective envelope of the Sun a smallscale ("intranetwork") component of the magnetic field, with a mean pressure of about $10 \%$ of the turbulent pressure,

$$
\frac{\left\langle B^{2}\right\rangle / 4 \pi}{\rho V_{\text {con }}^{2}} \equiv \varepsilon_{B} \sim 0.1 .
$$

This field is present even at solar minimum, when it is distributed almost uniformly across the solar disk (Murray 1992). It has been conjectured to be the consequence of a stochastic, nonhelical dynamo that builds up magnetic flux on the scale of an individual convective cell (TD93; Durney, de Young, \& Roxburgh 1993). Such a dynamo would operate almost independently of the global solar dynamo that manifests itself at the surface in the form of sunspot activity and the dipolar magnetic field. ${ }^{4}$ Some theoretical support for this conjecture comes from models of dynamo action in mirror symmetric turbulence (Ruzmaikin \& Sokoloff 1981; Meneguzzi \& Pouquet 1989).

It is now straightforward to estimate the equipartition magnetic field in the gain region. From equations (32) and (33),

$$
\begin{aligned}
\left\langle B^{2}\right\rangle^{1 / 2}= & \times 10^{13} \varepsilon_{B-1}^{1 / 2}\left(\frac{\dot{M}}{M_{\odot} \mathrm{s}^{-1}}\right)^{1 / 2} \\
& \times\left(\frac{R_{\mathrm{sh}}}{100 \mathrm{~km}}\right)^{-17 / 12}\left(\frac{\mathscr{R}}{10}\right)^{1 / 2}\left(\frac{\mathscr{L}_{52}}{4}\right)^{1 / 3} \\
& \times\left(\frac{M_{\text {core }}}{1.4 M_{\odot}}\right)^{3 / 4} \mathrm{G} .
\end{aligned}
$$

This field will be maintained in the gain region, against the downward flow, by buoyancy forces and neutrino heating (C. Thompson \& N. W. Murray, 2000, in preparation). For comparison, the equipartition magnetic field in the convective region (of radius $R_{\text {con }}$ ) inside the $v$-sphere is (DT92)

$$
\begin{aligned}
\left\langle B^{2}\right\rangle^{1 / 2}= & 3 \times 10^{15} L_{v 53}^{1 / 3} \varepsilon_{B-1}^{1 / 2}\left[\frac{M\left(<R_{\text {con }}\right)}{M_{\odot}}\right]^{1 / 6} \\
& \times\left(\frac{R_{\text {con }}}{20 \mathrm{~km}}\right)^{-7 / 6} \mathrm{G} .
\end{aligned}
$$

Here $L_{v}$ is the luminosity in all three flavors of neutrinos and antineutrinos. Although the magnetic field is weaker in the postshock layer, the density is also much lower there. After allowing for compression of the accreting material up

\footnotetext{
${ }^{4}$ The two dynamos are not, of course, entirely independent: the global dynamo feeds magnetic flux into the convection zone. However, one infers from the time variation of solar $p$-mode frequencies with solar cycle that the proportionality between magnetic and turbulent stresses is maintained down to least six pressure scale heights below the photosphere (Goldreich et al. 1991). This strongly indicates a local amplification mechanism that leads to a dynamical balance between the two stresses.
}

to nuclear matter density, the two convective layers are expected to yield magnetic fields of comparable strength (C. Thompson \& N. W. Murray, 2000, in preparation).

\subsection{Simultaneous Ram and Neutrino Heating}

Consider a bubble of magnetized fluid - or M-fluid for short — of density $\rho_{M}$ situated near the accretion shock. The outward buoyancy force acting on the bubble is

$$
\frac{(4 \pi / 3) a^{3}\left(\rho-\rho_{M}\right) g}{C_{D} \pi a^{2} \rho V_{R}^{2}} \simeq \frac{\rho-\rho_{M}}{\rho}\left(\frac{V_{R}^{2}}{2 G M_{\text {core }} / R_{\mathrm{sh}}}\right)^{-1}\left(\frac{a}{R}\right)
$$

relative to the (inward) accretion ram force caused by the ambient flow of density $\rho$ and speed $V_{R}$. At high Reynolds number, $C_{D} \simeq 1$. Just outside the shock, the accretion speed is $V_{R}^{2} \simeq 2 G M_{\text {core }} / R_{\text {sh }}$, and equation (36) implies that the ram force is the stronger by a factor $\sim(a / R)^{-1}$. Inside the shock, $V_{R}$ drops by a factor $\sim \mathscr{R}^{-1}$, and the relative strength of the buoyancy force increases by $\sim \mathscr{R}^{2}$. The bubble will remain pinned below the shock unless (or until) it is large enough to impede the accretion flow over a solid angle $\Delta \Omega \sim(a / R)^{2} \gtrsim 1$.

The shock adjacent to the bubble must be oblique to allow the flow to pass around the bubble (Fig. 1). Balancing the ram and buoyancy forces implies a flow speed

$$
V_{2}^{2} \sim\left(\frac{a}{R_{\mathrm{sh}}}\right)\left(\frac{\rho_{2}-\rho_{M}}{\rho_{2}}\right) \frac{2 G M_{\text {core }}}{R_{\mathrm{sh}}}
$$

below the shock. This speed can be expressed approximately in terms of the inclination angle $\theta$ between the shock normal and the radial direction,

$$
V_{2} \simeq \sin \theta \sqrt{\frac{2 G M_{\mathrm{core}}}{R_{\mathrm{sh}}}},
$$

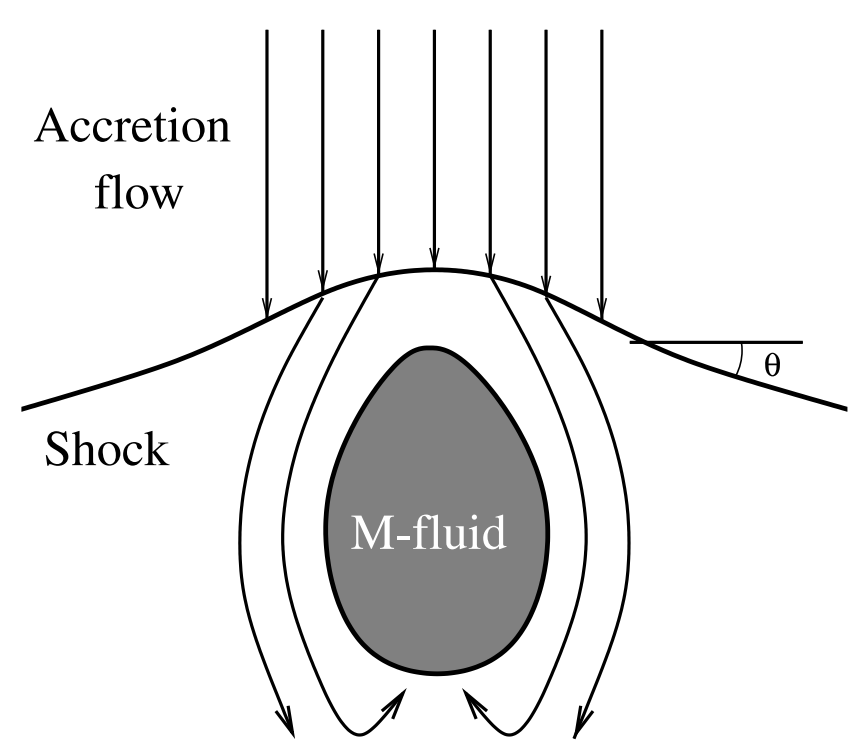

FIG. 1.-The buoyancy force pins a bubble of low-density M-fluid near the accretion shock. The corrugation of the shock adjusts to give enough downward postshock ram to balance the buoyancy force. The magnetic field is strongly amplified by the shear flow near the bubble boundary. Magnetized fluid that is advected downward becomes strongly buoyant in the hydrostatic atmosphere below the bubble, where the accretion speed decreases rapidly. 
when $\sin \theta \gg 1 / \mathscr{R}$. (Here $\mathscr{R}$ is the compression ratio across a normal shock.) The shock is corrugated through the rms angle

$$
\sin \theta \sim\left(\frac{a}{R_{\mathrm{sh}}}\right)^{1 / 2}\left(\frac{\rho_{2}-\rho_{M}}{\rho_{2}}\right)^{1 / 2} .
$$

As the bubble is compressible, the turbulent pressure in its interior approaches $\rho_{M} V_{2}^{2}$ (e.g., Batchelor 1970). These turbulent motions suffer negligible radiative damping on a scale $\sim \ell_{P}$. The optical depth to Compton scattering is enormous:

$$
\tau_{T}=2 a \sigma_{T} n_{e^{ \pm}}=6 \times 10^{13} T_{\mathrm{Mev}}^{3}\left(\frac{a}{10 \mathrm{~km}}\right)
$$

for a bubble of nondegenerate $e^{+}-e^{-}$plasma. Nonetheless, effective dissipation does result from a cascade to high wavenumber, where the wave motions are damped directly by Compton drag (Thompson \& Blaes 1998).

The equilibrium temperature in the bubble is obtained by balancing the heating rate $\sim \frac{1}{2} C_{D}\left(\pi a^{2}\right) \rho_{2} V_{2}^{3}$ over the crosssectional area of the bubble, against cooling throughout its volume. The density is assumed to be high enough that cooling is dominated by $e^{ \pm}$capture. Setting

$$
\rho_{2} V_{2}=(\mathscr{R} \sin \theta) \rho_{1} V_{1}
$$

from mass conservation, we obtain

$$
\frac{C_{D}}{2}\left(\pi a^{2}\right) \mathscr{R} \sin \theta\left(\frac{a}{R_{\mathrm{sh}}}\right)\left(\frac{\rho_{2}-\rho_{M}}{\rho_{2}}\right) \rho_{1} V_{1}^{3}=\dot{Q}^{e N} \frac{4 \pi}{3} a^{3} .
$$

The equilibrium temperature is, from equation (15),

$$
\begin{aligned}
T_{\text {eq }}= & 3.4 \sin ^{1 / 6} \theta\left(\frac{R_{\mathrm{sh}}}{100 \mathrm{~km}}\right)^{-5 / 12}\left(\frac{\rho_{2}}{\rho_{M}}-1\right)^{1 / 6} \\
& \times\left(\frac{M_{\text {core }}}{1.4 M_{\odot}}\right)^{1 / 4} \mathrm{MeV} .
\end{aligned}
$$

This result depends only on the density of the bubble relative to the accretion flow because both heating and cooling rates are proportional to density. At this equilibrium temperature, the pressure in relativistic particles is, relative to the accretion ram,

$$
\begin{aligned}
\frac{P_{e^{ \pm}}+P_{\gamma}}{\rho_{1} V_{1}^{2}}= & 1.8 \sin ^{2 / 3} \theta\left(\frac{\dot{M}}{M_{\odot} \mathrm{s}^{-1}}\right)^{-1}\left(\frac{R_{\mathrm{sh}}}{100 \mathrm{~km}}\right)^{5 / 6} \\
& \times\left(\frac{\rho_{2}}{\rho_{M}}-1\right)^{2 / 3}\left(\frac{M_{\text {core }}}{1.4 M_{\odot}}\right)^{1 / 2} \mathrm{MeV} .
\end{aligned}
$$

The right-hand side is generally larger than unity. Because $P_{e^{ \pm}}+P_{\gamma}$ cannot exceed the postshock pressure, ram heating causes the bubble to expand against the ambient pressure.

It is useful to know the fraction of the gravitational potential energy, liberated at the accretion shock, that is available for ram heating. This is, from equations (37) and (41),

$$
\begin{aligned}
\varepsilon_{\mathrm{ram}} & =\frac{1 / 2\left(C_{D} \rho_{2} V_{2}^{3}\right)}{1 / 2\left(\rho_{1} V_{1}^{3}\right)} \\
& =C_{D}(\mathscr{R} \sin \theta)\left(\frac{a}{R_{\mathrm{sh}}}\right)\left(\frac{\rho_{2}-\rho_{M}}{\rho_{2}}\right),
\end{aligned}
$$

assuming that the buoyant bubbles cover a large fraction of the area of the shock. A characteristic scale for individual bubbles is the pressure scale height, $2 a=\ell_{P} \simeq R / 4$, which implies

$$
\varepsilon_{\text {ram }} \simeq 0.1(\mathscr{R} \sin \theta) .
$$

The net (logarithmic) heating rate is then

$$
\begin{aligned}
t \dot{E}_{\mathrm{ram}}= & \frac{\varepsilon_{\mathrm{ram}} G M_{\mathrm{core}}(t \dot{M})}{R_{\mathrm{sh}}}=4 \times 10^{50}\left(\frac{\varepsilon_{\mathrm{ram}}}{0.1}\right) \\
& \times\left(\frac{t \dot{M}}{0.1 M_{\odot}}\right)\left(\frac{R_{\mathrm{sh}}}{100 \mathrm{~km}}\right)^{-1} \\
& \times\left(\frac{M_{\text {core }}}{1.4 M_{\odot}}\right) \mathrm{ergs} .
\end{aligned}
$$

In numerical simulations of Rayleigh-Taylor instabilities in highly conducting fluids, the magnetic field is strongly amplified in the shear layer separating the high- and lowdensity phases (Jun, Norman, \& Stone 1995). This suggests the dynamo mechanism illustrated in Figure 1. The flow speed first increases rapidly with radial distance below the oblique shock as the shocked fluid accelerates toward the free-fall velocity (at a large density contrast $\rho_{2} / \rho_{M}$ ). The magnetic field entrained in these elongated downflows is strongly sheared and stretched. However, below the layer of M-fluid, these downflows must decelerate to a speed $V_{1} / \mathscr{R} \simeq(1 / 10)\left(V_{1}\right)$ appropriate for a spherical shock. As a result, the buoyancy force acting on the magnetized fluid in the shear layer increases by $\sim 10-100$ relative to the downward ram force (eq. [37]). The increasing buoyancy of the M-fluid allows it to rise vertically and accumulate in the bubble interior. This circulatory motion recycles the magnetic field through the shear layer and, we conjecture, allows it to build up in a secular manner to a limiting pressure

$$
\frac{B^{2}}{8 \pi} \sim \rho_{M} V_{2}^{2} \sim \rho_{1} V_{1}^{2}\left(\frac{\mathscr{R}}{10}\right)\left(\frac{a}{0.1 R_{\mathrm{sh}}}\right)\left(1-\frac{\rho_{2}}{\rho_{M}}\right)
$$

that is comparable to the incident ram pressure.

Finally, let us compare the rate of neutrino heating of the M-fluid with the rate of ram heating (eq. [47]). Normalizing the M-fluid density $\rho_{M}$ to the postshock density (eq. [8]), the net heating rate within a shell of radial thickness $\ell_{P}=$ $\frac{1}{4} R_{\mathrm{sh}}$ is

$$
\begin{aligned}
t \dot{E}_{v N}= & \dot{Q}_{v N} \pi R_{\mathrm{sh}}^{3}=1.5 \times 10^{50}\left(\frac{\rho_{M}}{0.5 \rho_{2}}\right)\left(\frac{\mathscr{R}}{10}\right) \\
& \times\left(\frac{t \dot{M}}{0.1 M_{\odot}}\right)\left(\frac{R_{\mathrm{sh}}}{100 \mathrm{~km}}\right)^{-1 / 2} \\
& \times\left(\frac{\mathscr{L}_{52}}{4}\right)\left(\frac{M_{\text {core }}}{1.4 M_{\odot}}\right)^{-1 / 2} \text { ergs . }
\end{aligned}
$$

The ram heating rate (eq. [47]) is typically a few times larger. The reduction in density needed to prolong the accumulation of magnetic energy below the shock has the negative consequence of suppressing direct neutrino heating. However, the net heating rate $t\left(\dot{E}_{\text {ram }}+\dot{E}_{v N}\right)$ approaches $10^{51}$ ergs. The total energy released to the supernova shock is considered in the next section. 


\section{ACCRETIONAL HEATING AND ASYMMETRIC EXPANSION OF THE SHOCK}

We now examine the effectiveness of accretion in heating the supernova shock through the formation of a bubble of low-density fluid below the shock. Because buoyancy forces are induced by infall through the gravitational field of the neutron core, the expansion of the bubble will initially be quasi-static. We show that a spherical accretion shock overlying such a bubble is subject to a global RayleighTaylor mode, which allows its expansion to proceed anisotropically while accretion continues. This means that the energy of the supernova shock is set by the efficiency of accretional heating in the central core. We derive the equilibrium shape of an aspherical shock bounding a lowdensity, hydrostatic atmosphere and comment on the stability of this solution.

\subsection{Basic Energetic Requirements}

There are two basic energy scales in the problem: the energy released at the stalled accretion shock and the energy that must be expended to reverse the accretion flow and push the shock to infinity. In the present model, the two scales are distinct and simultaneously relevant. An asymmetric and quasi-static expansion of the shock allows accretion to continue from the part of the core diametrically opposite to the expanding plume. Accretion will continue (on the dynamical timescale) even after enough energy is accumulated to unbind the stellar mantle, and the shock begins to run outward. Given a density profile

$$
\rho_{0}\left(R_{0}\right) \simeq \mathscr{M} / R_{0}^{3}
$$

in the precollapse core, this hysteresis in the rate of accretion-driven heating allows the shock to accumulate a total energy

$$
\begin{aligned}
\Delta E \sim \varepsilon_{\mathrm{ram}} \frac{4 \pi G M_{\text {core }} \mathscr{M}}{R_{\mathrm{sh}}}=1.4 \times 10^{51}\left(\frac{\varepsilon_{\mathrm{ram}}}{0.1}\right) \\
\times\left(\frac{R_{\mathrm{sh}}}{50 \mathrm{~km}}\right)^{-1}\left(\frac{\mathscr{M}_{31}}{3}\right)\left(\frac{M_{\text {core }}}{1.4 M_{\odot}}\right) \mathrm{ergs} .
\end{aligned}
$$

From this perspective, an asymptotic shock energy of $\sim 10^{51}$ ergs reflects both the efficiency of conversion of gravitational binding energy to magnetic energy near the $v$-sphere and the density profile of the precollapse core.

Let us compare this energy with the minimum needed to push the shock to infinity. The material just exterior to the shock falls with ram pressure $\rho_{1} V_{1}^{2}$ (eq. [7]). The flow speed vanishes just below the shock (in the frame of the collapsed core) when the postshock pressure is increased by

$$
\Delta P=\frac{(\gamma+3)(\gamma-1)}{2(\gamma+1)}\left[\rho_{1} V_{1}^{2}\right]\left(R_{\mathrm{sh}}\right)
$$

over the value for a stationary shock (Bruenn 1993). This pressure increase allows the shock to move outward. Here $\gamma$ is the adiabatic index, $\gamma \simeq 4 / 3$ in the low-density M-fluid below the shock.

The accretion ram decreases with time even for a failed shock. The collapse time from initial radius $R_{0}$ to the central neutron core is given by $t \simeq 2\left(R_{0}^{3} / 2 G M_{\text {core }}\right)^{1 / 2}$, since $V_{R}$ in the outer parts of the collapse is about one-half of the free-fall speed (Yahil \& Lattimer 1982). This implies

$$
\begin{aligned}
\dot{M}(t) & =\frac{d M_{0}}{d R_{0}}\left(\frac{d t}{d R_{0}}\right)^{-1} \simeq \frac{8 \pi}{3} \frac{\mathscr{M}}{t} \\
& =1.2\left(\frac{t}{0.1 \mathrm{~s}}\right)^{-1}\left(\frac{\mathscr{M}_{31}}{3}\right) M_{\odot} \mathrm{s}^{-1} .
\end{aligned}
$$

At an enclosed mass of $1.75 M_{\odot}$, the mass parameter $\mathscr{M}$ ranges from $3 \times 10^{31} \mathrm{~g}$ for a $11 M_{\odot}$ progenitor to $\sim 10^{32} \mathrm{~g}$ for a 25-35 $M_{\odot}$ progenitor (WW95).

Substituting equation (53) for $M$ in equation (7) for the accretion ram, shows that the total energy input increases with shock radius $R_{\mathrm{sh}}$,

$$
\begin{aligned}
\Delta E & \simeq \frac{4 \pi}{3} R_{\mathrm{sh}}^{3} \frac{\Delta P}{\gamma-1} \\
& =\left[\frac{2^{5 / 2} \pi(\gamma+3)}{9(\gamma+1)}\right] \frac{\left(G M_{\mathrm{core}} R_{\mathrm{sh}}\right)^{1 / 2} \mathscr{M}}{t},
\end{aligned}
$$

at a fixed time $t$ following core bounce. The energy required to get a stalled shock moving outward through the accretion flow does not, in general, suffice to push the shock to infinity. When the shock succeeds in some numerical simulations (e.g., Wilson \& Mayle 1993; BHF; JM96), it generally does so by acquiring an excess of energy above the critical value in equation (54). Thus, a minimal estimate of the total energy input is obtained by setting $R_{\mathrm{sh}}$ equal to the radius $R_{R}$ of the rarefaction wave that marks the outer boundary of the collapsing core. The sound speed exterior to the rarefaction wave can be deduced from the equation of hydrostatic equilibrium together with the density profile in equation (50),

$$
c_{s}^{2}=\gamma \frac{P(R)}{\rho}=\gamma \frac{G M(<R)}{4 R} .
$$

Setting

$$
t=\int^{R_{R}} \frac{d R}{c_{s}(R)}
$$

and $\gamma=4 / 3$ yields

$$
\begin{aligned}
R_{R}(t) & =\frac{3^{1 / 3}}{2^{2 / 3}}\left[G M\left(<R_{R}\right) t^{2}\right]^{1 / 3} \\
& =2.3 \times 10^{3}\left[\frac{M\left(<R_{R}\right)}{1.4 M_{\odot}}\right]^{1 / 3}\left(\frac{t}{0.3 \mathrm{~s}}\right)^{2 / 3} \mathrm{~km} .
\end{aligned}
$$

The net binding energy of the material at this radius is

$$
\frac{G M\left(<R_{R}\right) \rho}{R_{R}}-\frac{P\left(R_{R}\right)}{\gamma-1}
$$

per unit volume. Integrating over the volume exterior to radius $R_{R}$ gives

$$
\begin{aligned}
E_{B} & =\left[1-\frac{1}{4(\gamma-1)}\right] \frac{4 \pi G M\left(<R_{R}\right) \mathscr{M}}{R_{R}} \\
& \simeq 1 \times 10^{50}\left[\frac{M\left(<R_{R}\right)}{1.4 M_{\odot}}\right]^{2 / 3}\left(\frac{\mathscr{M}_{31}}{3}\right)\left(\frac{t}{0.3 \mathrm{~s}}\right)^{-2 / 3} \mathrm{ergs} .
\end{aligned}
$$

The gravitational binding energy released by the infalling material increases with time, just as the binding energy in 
equation (59) continues to decrease. In general, only a small fraction,

$$
\begin{aligned}
{\left[1-\frac{1}{4(\gamma-1)}\right] \frac{R_{\mathrm{sh}}}{R_{R}(t)} } & \simeq 0.02\left(\frac{R_{\mathrm{sh}}}{100 \mathrm{~km}}\right) \\
& \times\left(\frac{M_{\text {core }}}{1.4 M_{\odot}}\right)^{-1 / 3}\left(\frac{t}{0.1 \mathrm{~s}}\right)^{-2 / 3},
\end{aligned}
$$

of the binding energy released at the accretion shock,

$$
\begin{aligned}
t \dot{E}_{\mathrm{acc}}= & \frac{G M_{\text {core }}}{R_{\mathrm{sh}}} t \dot{M}=\left(\frac{8 \pi}{3}\right) \frac{G M_{\text {core }} \mathscr{M}}{R_{\mathrm{sh}}} \\
= & 5 \times 10^{51}\left(\frac{M_{\text {core }}}{1.4 M_{\odot}}\right)\left(\frac{\mathscr{M}_{31}}{3}\right) \\
& \times\left(\frac{R_{\mathrm{sh}}}{100 \mathrm{~km}}\right)^{-1} \text { ergs },
\end{aligned}
$$

need be expended to push the outer shock to infinity. The actual efficiency of ram heating (eq. [45]) is estimated to be higher than this minimum value.

The binding energy, measured at the cut between accreted and ejected mass, tends to be larger in the heavier progenitors (WW95). This trend is compensated by an increase in the rate of accretional heating, which is also proportional to $\mathscr{M}$. As a result, the explosion energy may in fact increase with progenitor mass. Failure of the supernova remains a possibility if the central neutron core has collected enough mass to collapse to a black hole before the outer shock succeeds (see Brown \& Bethe 1994).

\subsection{Conditions for Quasi-static and Asymmetric Expansion of an Accretion Shock}

The accretion flow through a failed shock onto the neutron core has several distinct properties that should be summarized before proceeding further. Unlike most astrophysical accretion flows, which are channeled through a centrifugally supported disk, it is essentially spherical during its first stages; a low-density component of the flow has no easy path of escape. In contrast with accretion onto a protostar, the flow is optically thin to the dominant carrier of radiative energy (neutrinos). As a result, a buoyant component of the flow cools more slowly than it is heated by the incident accretion ram $(\S 3.2)$. Accretion onto the neutron core continues for $\sim 100$ dynamical times $\left(R_{\mathrm{sh}}^{3} / 2 G M_{\text {core }}\right)^{1 / 2}=0.002\left(R_{\mathrm{sh}} / 100 \mathrm{~km}\right)^{1 / 2}\left(M_{\text {core }} / 1.4 M_{\odot}\right)^{-1 / 2}$ $\mathrm{s}$. This allows the secular accumulation of energy below the shock.

It is also instructive to contrast this situation with hierarchical clustering in an expanding universe. An isolated mass concentration such as a galaxy or galaxy cluster grows rapidly for a much smaller number of dynamical times before being subsumed into a larger structure. Accretion of hot gas onto the outer parts of a virialized cluster proceeds adiabatically, which forces the accretion shock to expand with the growing cluster. During this expansion, a magnetic field amplified by shear stresses near the shock can be expected to accumulate an approximately constant fraction of the internal energy in the region below the shock. As a result, the accretional energy is spread over a progressively larger volume. The situation in a supernova core is quite different: the failed bounce shock remains almost stationary as the accretion flow continues over many dynamical times. It is this combination of sustained, spherical accretion and slow cooling in the vicinity of the shock that, we conjecture, allows the secular accumulation of energy in a low-density, magnetized fluid.

We should emphasize that this process is self-regulating because the heating rate is directly proportional to the accretion rate onto the central neutron core. In the neutrinoheated shock model - and other models in which the central energy source is partly or wholly decoupled from the accretion flow- the shock moves outward too rapidly for low harmonic Rayleigh-Taylor modes to develop (Chevalier \& Klein 1978; Goodman 1990).

Now let us consider the transport of low-density, magnetized fluid (M-fluid) from the vicinity of the accretion shock out to the rarefaction wave that bounds the central collapsing core. An individual bubble of M-fluid is contained by the accretion ram until its angular cross section grows to $\Delta \Omega_{M} \sim 1$ (eq. [36]). The outward motion of a such a large bubble will deform the accretion flow. Rapid heating by the accretion ram requires that the accretion flow continue unimpeded in one hemisphere, i.e., that the bubble expand anisotropically and quasi-statically. The internal pressure near the outer radius $R_{M}$ of the bubble is $P_{M} \sim$ $\rho_{1}\left(R_{M}\right) V_{1}^{2}\left(R_{M}\right)$. Such a quasi-static expansion is possible as long as the energy injected into the bubble over the dynamical time $\left(R_{M}^{3} / 2 G M_{\text {core }}\right)^{1 / 2}$ is less than, or comparable to, its total internal energy $E_{M}=\frac{1}{3} R_{M}^{3} \Delta \Omega_{M}\left(3 P_{M}\right) \propto R_{M}^{1 / 2}$.

To make this comparison, we must first account for the effects of adiabatic expansion. The energy increment $\Delta E_{M}$ of the bubble is related to the injected energy $\Delta E_{0}$ by

$$
\Delta E_{M}=\Delta E_{0}-\delta_{M} \frac{E_{M}}{R_{M}} \Delta R_{M} .
$$

Uncompensated adiabatic losses in a bubble with adiabatic index $\gamma_{M}=4 / 3$ correspond to $\delta_{M}=1$. These losses involve work done by the expanding bubble on the accretion flow. Some of this energy will be returned: for example, the expanding magnetic field in the bubble is sheared and tangled by its interaction with denser Rayleigh-Taylor plumes of accreting material flowing through the edges and interior of the bubble. As long as the bubble expands quasistatically, this equation is supplemented by

$$
\frac{1}{E_{M}} \frac{d E_{M}}{d t}=\frac{1}{2 R_{M}} \frac{d R_{M}}{d t}
$$

and one finds

$$
\frac{d E_{M}}{d t}=\frac{1}{1+2 \delta_{M}} \frac{d E_{0}}{d t} .
$$

For example, $d E_{M} / d t=\frac{1}{2} d E_{0} / d t$ if the compensated adiabatic losses are half the uncompensated value $\left(\delta_{M}=\frac{1}{2}\right)$. We conclude that adiabatic losses are not likely to cut the heating efficiency by a large factor.

Energy is injected into the bubble at a rate

$$
\frac{d E_{0}}{d t}=\varepsilon_{\mathrm{ram}} \frac{G M_{\mathrm{core}} \dot{M}}{R_{\mathrm{sh}}}
$$

so quasi-static expansion remains possible out to a radius where

$$
\begin{aligned}
\frac{\varepsilon_{\mathrm{ram}}}{1+2 \delta_{M}} & \frac{G M_{\mathrm{core}} \dot{M}}{R_{\mathrm{sh}}}\left(\frac{R_{M}^{3}}{2 G M_{\mathrm{core}}}\right)^{1 / 2} \\
\simeq & \rho_{1}\left(R_{M}\right) V_{1}^{2}\left(R_{M}\right) R_{M}^{3} \Delta \Omega_{M} .
\end{aligned}
$$


This critical outer radius, denoted by $R_{\exp }$, is

$$
\frac{R_{\mathrm{exp}}}{R_{\mathrm{sh}}}=\frac{2\left(1+2 \delta_{M}\right)}{\varepsilon_{\mathrm{ram}}}\left(\frac{\Delta \Omega_{M}}{4 \pi}\right) \simeq 20\left(\frac{\varepsilon_{\mathrm{ram}}}{0.1}\right)^{-1}\left(\frac{\Delta \Omega_{M}}{2 \pi}\right) .
$$

Beyond this radius, the expansion velocity of the plume becomes comparable to its internal sound speed, and the shock that bounds it begins to run outward and sideways. The net effect is an explosion offset a distance $\sim \frac{1}{2} R_{\text {exp }}$ from the neutron core.

Effective seeding of the M-fluid below the shock requires a finite magnetic field. An underdense parcel of fluid is advected downward from the accretion shock if it is smaller than $a / R \sim \mathscr{R}^{-2}$ (eq. [36]). The required seed field is easily provided by the strongly convective layers in the gain region below the shock and in the central neutron core. Estimates of the equilibrium magnetic fields in each of these regions are given by equations (34) and (35).

\subsection{Stability of a Spherical Accretion Shock}

The expansion of a low-density bubble through a supersonic accretion flow can be approached self-consistently in the following way. Consider a spherically symmetric shock overlying a shell of low-density fluid, which in turn overlies the neutron core (Fig. 2). The sound speed in the lowdensity shell is assumed to be high enough that its pressure $P_{M}$ is essentially constant. Now consider a slow, nonradial perturbation of the accretion shock that preserves the volume and internal energy of the shell, e.g., $\delta R_{\mathrm{sh}}(\theta)=$ $\delta R_{\mathrm{sh}, 0} \cos \theta$. Where the shock moves outward $(\pi / 2$ $>\theta>0$ ), the accretion ram drops below $P_{M}$ and cannot resist further expansion of the shock. Conversely, the accretion ram overcomes the internal pressure of the low-density shell where the shock moves inward. ${ }^{5}$

This instability is a generalization of the classic RayleighTaylor instability, with the shock replacing the interface between the two fluids, and the accretion ram force replacing the hydrostatic force of the upper, denser fluid. In contrast to the usual Rayleigh-Taylor criterion, instability is possible even if the fluid upstream of the shock is less dense than the fluid below the shock. A simple criterion for marginal stability, which includes the effects of compressibility in the fluid below the shock, may be obtained as follows. The shocked fluid is approximated as a thin shell adjacent to the shock (Fig. 2). The thickness of the shocked shell depends on the effectiveness with which high-wavenumber Rayleigh-Taylor modes allow the shocked fluid to pass through the lighter fluid below. Thus, the dynamics may be divided into two regimes: the thin-shell regime, where the shell mass $M_{\mathrm{sh}}$ is much smaller than

$$
\tilde{M}_{\mathrm{sh}} \equiv \dot{M}\left(\frac{R_{\mathrm{sh}}^{3}}{2 G M_{\mathrm{core}}}\right)^{1 / 2}=4 \pi \rho_{1} R_{\mathrm{sh}}^{3} \propto R_{\mathrm{sh}}^{3 / 2}
$$

and the thick-shell regime, where $M_{\mathrm{sh}}$ is much larger than $\tilde{M}_{\text {sh }}$.

The perturbation $\delta P_{M}$ to the pressure at the outer boundary of the low-density bubble (immediately below the

\footnotetext{
${ }^{5}$ The component of the accretion velocity perpendicular to the shock is first order in $\delta R_{\mathrm{sh}}$ but only second order in the angular gradient $d R_{\mathrm{sh}} / d \theta=$ $-\delta R_{\mathrm{sh}} \tan \theta$.
}

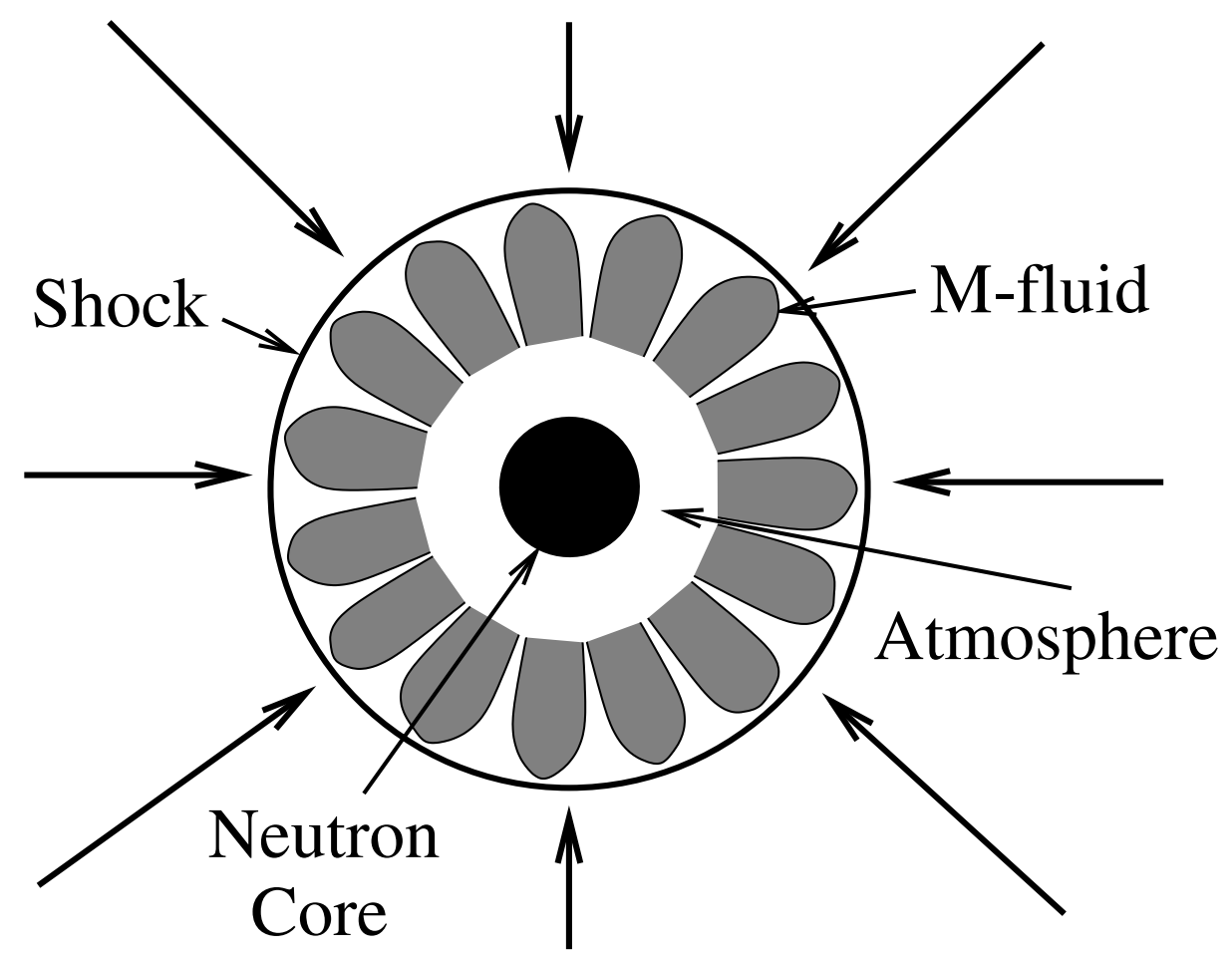

FIG. 2.-A region of low-density M-fluid sandwiched between a failed accretion shock and the neutron core. A thin layer of shocked material drains into dense Rayleigh-Taylor filaments, which, in turn, feed the deleptonizing atmosphere of the neutron core. 
shocked shell) is

$$
\begin{aligned}
\delta P_{M}= & \delta\left(\rho_{1} V_{1}^{2}+\frac{G M_{\mathrm{core}} M_{\mathrm{sh}}}{4 \pi R_{\mathrm{sh}}^{4}}\right) \\
= & -\left(\frac{5}{2} \rho_{1} V_{1}^{2}+\frac{5}{2} \frac{G M_{\mathrm{core}} M_{\mathrm{sh}}}{4 \pi R_{\mathrm{sh}}^{4}}\right) \frac{\delta R_{\mathrm{sh}}}{R_{\mathrm{sh}}} \\
& +2 \rho_{1} V_{1} \frac{\partial\left(\delta R_{\mathrm{sh}}\right)}{\partial t},
\end{aligned}
$$

assuming $M_{\text {sh }}$ to remain a constant fraction of $\tilde{M}_{\text {sh }}$. Evaluating $\delta P_{M}$ in the low-density bubble itself, one finds two contributions,

$$
\begin{aligned}
\delta P_{M} & =\delta P_{M}\left(R_{\mathrm{sh}}\right)+\frac{\partial P_{M}}{\partial R}\left(R_{\mathrm{sh}}\right) \delta R_{\mathrm{sh}} \\
& =\delta P_{M}\left(R_{\mathrm{sh}}\right)-\rho_{M} \frac{G M_{\text {core }}}{R_{\mathrm{sh}}^{2}} \delta R_{\mathrm{sh}} .
\end{aligned}
$$

The first term, $\delta P_{M}\left(R_{\mathrm{sh}}\right)$, is related directly to the acceleration of the bubble boundary through the Euler equation

$$
\frac{\partial^{2} \delta R_{\mathrm{sh}}}{\partial t^{2}}=-\frac{1}{\rho_{M}} \frac{\partial \delta P_{M}}{\partial R}-\frac{\delta \rho_{M}}{\rho_{M}} \frac{G M_{\text {core }}}{R_{\mathrm{sh}}^{2}} .
$$

One must be careful to distinguish the density $\rho_{M}$ in the low-density bubble from the density $\rho_{2}$ immediately below the accretion shock (eq. [8]).

All the perturbed variables are given a harmonic time dependence $e^{-i \omega t}$. At marginal stability, $\omega=0$ and equation (71) implies that $\delta P_{M}\left(R_{\mathrm{sh}}\right)$ vanishes. ${ }^{6}$ Combining equations (69) and (70) then gives the criterion for marginal stability,

$$
\rho_{M}=\frac{5}{2}\left(\frac{\rho_{1} V_{1}^{2}}{G M_{\text {core }} / R_{\mathrm{sh}}}\right)+\frac{5 M_{\mathrm{sh}}}{8 \pi R_{\mathrm{sh}}^{3}}=5\left(1+\frac{M_{\mathrm{sh}}}{2 \tilde{M}_{\mathrm{sh}}}\right) \rho_{1} .
$$

In the thin-shell regime $\left(M_{\mathrm{sh}} \simeq 0\right)$, this critical density is about a factor of 2 smaller than the density immediately below a spherical accretion shock (taking into account the increase in the compression ratio because of photodissociation; eq. [5]). However, instability probably requires a larger density contrast than implied by equation (72): the infalling Rayleigh-Taylor fingers of shocked fluid must fill a small fraction of the volume in order to have a negligible effect on the stratification of the low-density fluid.

Strong instability at low-order spherical harmonics requires that the shock remain quasi-static. An expanding shock exhibits Rayleigh-Taylor modes but only at higher orders. Examples include the outer boundary of a pulsardriven bubble that expands through a wind with a steep density profile (Jun 1998) and the contact discontinuity separating a heavy shell of supernova ejecta from a decelerating forward shock (Chevalier, Blondin, \& Emmering 1992). In a decelerating supernova shell, the ram pressure of the ejecta passing through the reverse shock increases with the distance upstream of the reverse shock (e.g., toward the center of the explosion). In that situation, our analysis implies stability at low-order harmonics.

The growth rate away from marginal stability is derived in the Appendix in two regimes. In the thin-shell regime, the

\footnotetext{
${ }^{6}$ As is shown explicitly in the Appendix, $\delta P_{M}$ and $\partial\left(\delta P_{M}\right) / \partial R$ both vanish at radius $R_{\mathrm{sh}}$ at marginal stability.
}

dispersion relation close to marginal stability is

$$
\begin{aligned}
\frac{\omega^{2}}{G M_{\mathrm{core}} / R_{\mathrm{sh}}^{3}}= & {\left[\frac{\rho_{M}}{\rho_{1}}-5-i 2 \sqrt{2}\left(\frac{\omega}{\sqrt{G M_{\mathrm{core}} / R_{\mathrm{sh}}^{3}}}\right)\right] } \\
& \times\left[\frac{3}{8}-(2-\sqrt{3}) \frac{\rho_{1}}{\rho_{M}}\right]
\end{aligned}
$$

for $\ell=1$; and

$$
\begin{aligned}
\frac{\omega^{2}}{G M_{\mathrm{core}} / R_{\mathrm{sh}}^{3}}= & {\left[\frac{\rho_{M}}{\rho_{1}}-5-i 2 \sqrt{2}\left(\frac{\omega}{\sqrt{G M_{\mathrm{core}} / R_{\mathrm{sh}}^{3}}}\right)\right] } \\
& \times\left[\frac{3}{8}+\left(\ell-\frac{\Gamma}{2 \Gamma-1}\right) \frac{\rho_{1}}{\rho_{M}}\right]
\end{aligned}
$$

for $\ell \gg 1$. By contrast, the system is guaranteed to be far from marginal stability in the thick-shell regime, and the dispersion relation is

$$
\frac{\omega^{2}}{G M_{\text {core }} / R_{\text {sh }}^{3}}=-\frac{1}{2}[1+4 \ell(\ell+1)]^{1 / 2}+\frac{1}{2} .
$$

This frequency is imaginary for all positive values of $\ell$.

\subsection{Equilibrium Shape of the Shock}

The equilibrium shape of the shock is easily calculated when $\ell_{P} \gg R$ and the pressure takes a constant value $P_{M}$ inside the Rayleigh-Taylor plume of M-fluid. The material below the shock remains in hydrostatic equilibrium as long as the outer radius of the shock $R_{M}$ is smaller than in equation (67). Since the Atwood number $\left(\rho-\rho_{M}\right) /\left(\rho+\rho_{M}\right)$ is close to unity, shocked material accumulating above the lower density bubble descends in narrow, Rayleigh-Taylor spikes that fill only a fraction $\sim \mathscr{R}^{-1}$ of the volume. The shape of the shock is then expected to be insensitive to the spatial distribution of the dense, shocked material. Conservation of the flow of momentum perpendicular to the shock constrains the inclination angle $\alpha$ between the shock normal and the radial direction (Fig. 3),

$$
\cos \alpha=\frac{P_{M}}{\rho_{1}\left(R_{\mathrm{sh}}\right) V_{1}^{2}\left(R_{\mathrm{sh}}\right)}=\left(\frac{R_{\mathrm{sh}}}{R_{M}}\right)^{5 / 2} \quad\left(R \leq R_{M}\right) .
$$

The radius of the shock varies with polar angle $\theta$ (where $\theta=0$ at $R_{\mathrm{sh}}=R_{M}$ ) according to $R_{\mathrm{sh}}^{-1} d R_{\mathrm{sh}} / d \theta=\tan \alpha$. This equation integrates to

$$
\left(\frac{R_{\mathrm{sh}}}{R_{M}}\right)^{5 / 2}=\cos \left(\frac{5}{2} \theta\right)
$$

This solution collapses to small radius at $\theta=\pi / 5$.

The volume of low-density M-fluid is bounded below by a hydrostatically supported and quasi-spherical atmosphere. The solution from equation (77) must therefore join smoothly with a nearly spherical shock, at a radius $R_{\text {sphere }}$ close to the position of the failed bounce shock. Because the accretion ram decreases with radius as $R^{-5 / 2}$, the internal pressure can balance the accretion ram both at the outer shock radius $R_{M}$ and the inner shock radius $R_{\text {sphere }}$ only if the region below the base of the M-fluid (at $R<R_{\text {base }}$ ) is strongly stratified. A natural choice is the adiabatic profile $P(R) \propto R^{-4}$ from $R_{\text {sphere }}$ out to $R_{\text {base }}$ since neutrino heating is rapid enough to force convective motions from the gain 


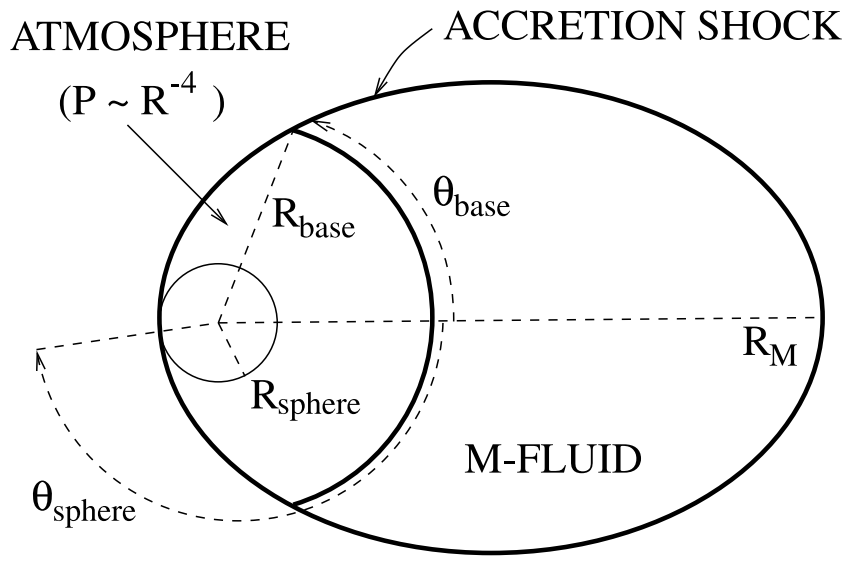

FIG. 3.-A large volume of M-fluid forces an asymmetric RayleighTaylor extension of the accretion shock from a minimum radius $R_{\text {sphere }}$ (the original radius of the failed shock) out to a maximum radius $R_{M}$. The accretion flow outside the shock converges spherically on the neutron core (toward the center of the circle of radius $R_{\text {sphere }}$ ). The M-fluid between radius $R_{\text {base }}$ and radius $R_{M}$ has a higher entropy than the atmosphere below $R_{\text {base }}$. Both zones are assumed to be spherically stratified and adiabatic, with the lower zone being a continuous upward extension of the convective atmosphere below radius $R_{\text {sphere }}$.

radius (below $R_{\text {sphere }}$ ) out to $R_{\text {base }}$ (Fig. 3). At $R_{\text {sphere }}<$ $R_{\mathrm{sh}}<R_{\text {base }}$, the equation for the shock tilt becomes

$$
\cos \alpha=\left(\frac{R_{\text {sh }}}{R_{\text {sphere }}}\right)^{-3 / 2}
$$

This integrates to give

$$
\left(\frac{R_{\text {sh }}}{R_{\text {sphere }}}\right)^{-3 / 2}=\cos \left[\frac{3}{2}\left(\theta_{\text {sphere }}-\theta\right)\right],
$$

where $\theta_{\text {sphere }}$ is the polar angle at which the shock reaches radius $R_{\text {sphere }}$. At radius $R_{\text {base }}$ (angle $\theta_{\text {base }}$ ), this equation matches onto equation (77).

Continuity of pressure at radius $R_{\text {base }}$ implies

$$
\left(\frac{R_{\text {base }}}{R_{\text {sphere }}}\right)=\left(\frac{R_{M}}{R_{\text {sphere }}}\right)^{5 / 8} \text {. }
$$

As a result, the angles $\theta_{\text {sphere }}$ and $\theta_{\text {base }}$ are related by $\cos \left[5 / 2\left(\theta_{\text {base }}\right)\right]=\cos \left[3 / 2\left(\theta_{\text {sphere }}-\theta_{\text {base }}\right)\right]$ or, equivalently,

$$
\theta_{\text {sphere }}=\frac{8}{3} \theta_{\text {base }} \text {. }
$$

Combining this equation with equations (77) and (79) gives the following expression for $\theta_{\text {base }}$ in terms of the maximum shock radius,

$$
\cos \left(\frac{5}{2} \theta_{\text {base }}\right)=\left(\frac{R_{M}}{R_{\text {sphere }}}\right)^{-15 / 16} .
$$

When $R_{M} \gg R_{\text {sphere }}$, this yields

$$
\theta_{\text {base }}=\frac{\pi}{5} ; \quad \theta_{\text {sphere }}=\frac{8 \pi}{15} .
$$

The interior of the plume will, more realistically, be stratified. Neutrino heating at its base is strong enough to drive convection ( $(2.3)$, as is observed in numerical models of spherical, neutrino-heated shocks (Herant et al. 1992; BHF; JM96). The entropy is high enough that radiation and pairs dominate the pressure. Approximating the adiabatic index ${ }^{7}$ by $\gamma=4 / 3$, the equation of hydrostatic equilibrium integrates to

$$
\frac{P(R)}{\rho_{M}(R)}-\frac{P\left(R_{M}\right)}{\rho_{M}\left(R_{M}\right)}=\frac{G M_{\text {core }}}{4}\left(\frac{1}{R}-\frac{1}{R_{M}}\right)
$$

at constant entropy. Substituting equation (76) at $R=R_{M}$ and $V_{1}^{2}\left(R_{M}\right)=2 G M_{\text {core }} / R_{M}$, we obtain

$$
\frac{P(R)}{P\left(R_{M}\right)}=\left[1+\frac{\rho_{M}\left(R_{M}\right)}{8 \rho_{1}\left(R_{M}\right)}\left(\frac{R_{M}}{R}-1\right)\right]^{4} \quad\left(R \leq R_{M}\right) .
$$

An upper bound to the M-fluid density at radius $R_{M}$ is obtained by requiring that the pressure at radius $R_{\text {sphere }}$ be no smaller than the accretion ram $\left[\rho_{1} V_{1}^{2}\right]\left(R_{\text {sphere }}\right)$ at that radius,

$$
\begin{aligned}
\left.\rho_{M}\left(R_{M}\right)\right|_{\max } & =8 \rho_{1}\left(R_{M}\right) \frac{\left(R_{M} / R_{\text {sphere }}\right)^{5 / 8}-1}{\left(R_{M} / R_{\text {sphere }}\right)-1} \\
& \simeq 8 \rho_{1}\left(R_{M}\right)\left(\frac{R_{\text {sphere }}}{R_{M}}\right)^{3 / 8} \quad\left(R_{\text {sphere }} \ll R_{M}\right) .
\end{aligned}
$$

Thus, the density contrast between the M-fluid and the shocked accretion flow is guaranteed to be high. The lower boundary of the $\mathrm{M}$-fluid sits at a radius

$$
\begin{aligned}
\frac{R_{\text {base }}}{R_{\text {sphere }}} & =X^{5 / 8}\left[\frac{(X-1)-\left(\rho_{M} /\left.\rho_{M}\right|_{\max }\right)\left(X-X^{3 / 8}\right)}{(X-1)-\left(\rho_{M} /\left.\rho_{M}\right|_{\max }\right)\left(X^{5 / 8}-1\right)}\right], \\
X & \equiv \frac{R_{M}}{R_{\text {sphere }}},
\end{aligned}
$$

which approaches equation (80) when $\rho_{M}\left(R_{M}\right) \ll$ $\left.\rho_{M}\left(R_{M}\right)\right|_{\max }$.

The profile of the shock bounding the Rayleigh-Taylor plume is shown in Figure 4 for various values of $\rho_{M}\left(R_{M}\right) /\left.\rho_{M}\left(R_{M}\right)\right|_{\max }$. When the M-fluid density is close to its maximum value, the shock wraps almost $180^{\circ}$ around the star before meeting the spherical shock segment at radius $R_{\text {sphere }}$. Note that the accretion flow near the base of the shock (radius $R_{\text {sphere }}$ ) is strongly sheared.

The entropy of the plume is higher than the entropy of the accretion flow that sits below radius $R_{\text {sphere }}$. However, if $\rho_{M}\left(R_{M}\right)$ is comparable to $\left.\rho_{M}\left(R_{M}\right)\right|_{\max }$, then the (radiation) entropy per baryon is not much higher:

$$
\frac{\left[P^{3 / 4} / \rho_{M}\right]\left(R_{M}\right)}{\left[P^{3 / 4} / \rho_{2}\right]\left(R_{\text {sphere }}\right)}=\left(\frac{\mathscr{R}}{8}\right)\left(\frac{\rho_{M}\left(R_{M}\right)}{\left.\rho_{M}\left(R_{M}\right)\right|_{\max }}\right)^{-1} .
$$

Here, $\mathscr{R} \sim 10$ is the compression ratio across the shock at radius $R_{\text {sphere }}, \rho_{2}=\mathscr{R} \rho_{1}\left(R_{\text {sphere }}\right)$, and we make use of the asymptotic form of equation (86) appropriate to small $R_{\text {sphere }} / R_{M}$. When $\rho_{M}\left(R_{M}\right)<\left.\rho_{M}\left(R_{M}\right)\right|_{\max }$ there is a radial entropy jump at radius $R_{\text {base }}$, which permits the upward passage of low-density M-fluid, but not weakly magnetized fluid.

\subsection{Stability of an Asymmetric Accretion Shock}

A static, spherical shock sitting in an accretion flow suffers from a global Rayleigh-Taylor mode, if the volume below the shock is dominated by a fluid of density $\rho_{M} \lesssim$ $\frac{1}{2} \mathscr{R} \rho_{1}$ (eq. [72]). In pressure equilibrium, the (radiation) entropy of this low-density fluid exceeds the entropy imme-

\footnotetext{
${ }^{7}$ Dissociation of $\alpha$-particles at $T \sim 1 \mathrm{MeV}$ causes the largest departure from constant $\gamma$.
} 


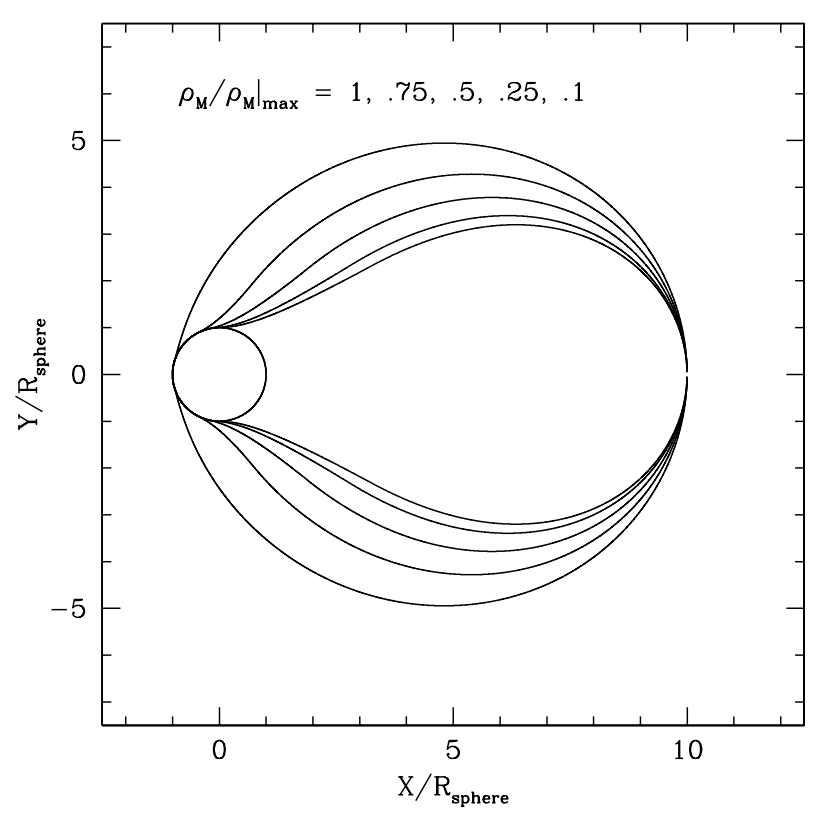

FIG. 4.- Shape of a quasi-static accretion shock surrounding a spherically stratified and isentropic plume of low-density M-fluid (eqs. [77], [79]). The various shapes correspond to different $M$-fluid densities (entropies), with the outermost curve having the largest density.

diately below the shock by a factor $\gtrsim 2$. The existence of asymmetric, static shock solutions at lower entropies begs the question as to whether these solutions with $\rho_{M}\left(R_{M}\right) \sim$ $\left.\rho_{M}\left(R_{M}\right)\right|_{\max }$ are stable to collapse. When the RayleighTaylor plume is very extended $\left(R_{M} \gg R_{\text {sphere }}\right)$, the entropy of the M-fluid is far higher than the entropy of the fluid that is shocked near the outer radius $R_{M}$; and its density is far lower (eq. [86]). If the shock were to relax to a spherical shape while maintaining pressure balance with the accretion ram, it would lie far outside the original stall radius $R_{\text {sphere }}$. It would still be unstable according to the criterion in equation (72). This indicates that an asymmetric Rayleigh-Taylor plume will maintain itself at a lower entropy than is required for its formation, after the plume has expanded to $R_{M} \gg R_{\text {sphere }}$.

Another simple argument suggests that this RayleighTaylor plume is stable to division into smaller units. Individual bubbles of angular size $\Delta \Omega_{M}<1$ are swept back by the accretion flow (eq. [36]). The energy accumulated by the M-fluid must increase with radius (as $\sim R_{M}^{1 / 2}$ ) in order to maintain $\Delta \Omega_{M} \sim 1$ (eq. [54]).

Direct neutrino cooling of the M-fluid becomes less effective as the Rayleigh-Taylor plume grows. By contrast, the energy input from below remains approximately constant (assuming an unimpeded accretion flow in the hemisphere opposite to the plume). The temperature of the M-fluid decreases as $T_{M} \propto R_{M}^{-5 / 8}$, so the contribution to the neutrino cooling rate from the outer parts of the plume decreases with $R_{M}$ as

$$
\dot{Q}^{e^{ \pm}} R_{M}^{3} \propto T_{M}^{6} \rho_{M} R_{M}^{3} \propto R_{M}^{-15 / 4} .
$$

This scaling presupposes that cooling is dominated by $e^{ \pm}$ capture and that the mass of the plume is conserved. If, instead, $\rho_{M}$ remains a constant fraction of the local density of the accretion flow, then $\rho_{M} R_{M}^{3} \propto R_{M}^{3 / 2}$ and $\dot{Q}^{e \pm} \propto R_{M}^{-9 / 4}$.

\section{IMPLICATIONS FOR PULSAR ROTATION AND PROPER MOTIONS}

The distribution of initial spin periods of isolated neutron stars is not yet well determined. Nonetheless, the distribution of measured spins of neutron stars that are associated with supernova remnants $\left(t_{\mathrm{SNR}}<10^{4} \mathrm{yr}\right)$ is strongly bimodal. The measured values are either less than $\sim 0.1 \mathrm{~s}$ (radio pulsars; e.g., Kulkarni 1992); or greater than $\sim 5 \mathrm{~s}$ (candidate "magnetars" such as the Soft Gamma Repeater and Anomalous X-ray Pulsar sources; e.g., Frail 1998). Selection effects could severely limit the detectability of neutron stars of intermediate spin periods since they are expected to be both weaker radio sources and (in the magnetar model) weaker X-ray sources caused by lower magnetic dissipation. These facts are, nonetheless, consistent with the hypothesis that all neutron stars are born rapidly rotating $(P<0.1 \mathrm{~s})$, if one accepts the further theoretical postulate that magnetars are formed as the result of largescale, helical dynamo action at initial spin periods in the millisecond range (DT92). Such a high frequency of rapid initial spin rates is puzzling at first sight and surely provides an important clue to the supernova mechanism.

The distribution of (three-dimensional) proper motions averages $\sim 400-500 \mathrm{~km} \mathrm{~s}^{-1}$, according to Lyne \& Lorimer (1994). A lower mean proper motion of $250-300 \mathrm{~km} \mathrm{~s}^{-1}$ was deduced by Hansen \& Phinney (1997) as the result of an apparent correlation between observed proper motion and radio luminosity. Cordes \& Chernoff (1998) found a similar result to Lyne \& Lorimer, but their analysis strongly favors at least two components to the velocity distribution, one of which dominates the pulsar population and has a lower mean proper motion of $\sim 300 \mathrm{~km} \mathrm{~s}^{-1}$. Fryer, Burrows, \& Benz considered both binary neutron star systems and isolated pulsars and found a mean proper motion of 400-500 $\mathrm{km} \mathrm{s}^{-1}$. There is evidence that two soft gamma repeaters (SGR 0526-66 and SGR 1900+14) have large proper motions of the order of $1000 \mathrm{~km} \mathrm{~s}^{-1}$ (DT92; Vasisht et al. 1994; Kouveliotou et al. 1999), but it is premature to say that this is a general characteristic of highly magnetized neutron stars. SGR 1806-20 may either be bound gravitationally to a highly luminous Be/LBV star (Van Kerkwijk et al. 1995) or be unbound but have a relatively small proper motion of $\sim 100 \mathrm{~km} \mathrm{~s}^{-1}$ (Hurley et al. 1999). The beststudied anomalous X-ray pulsar 1E 2259+586 lies fairly close to the center of the associated SNR CTB 109 (e.g., Fahlman \& Gregory 1981), as does 1E 1841-045 relative to Kes 73 (Gotthelf \& Vasisht 1997). Further evidence for diversity comes from the detection of radio pulsars with ordinary magnetic dipole fields and with proper motions approaching $1000 \mathrm{~km} \mathrm{~s}^{-1}$ (Frail \& Kulkarni 1991; Lyne \& Lorimer 1994). Large proper motions are not obviously tied to strong magnetism, which suggests that more than one physical mechanism may contribute to neutron star proper motions.

The high proper motions of radio pulsars have been ascribed (1) to asymmetries in the precollapse core (Goldreich \& Weber 1980); (2) to large-scale convective motions in between the $v$-sphere and the shock (Janka \& Mueller 1994; Burrows \& Hayes 1996); or (3) to asymmetric neutrino emission, driven directly by convective stresses (Woosley 1987), by magnetic stresses amplified by convection (TD93) or by asymmetric neutrino absorption and scattering caused by spin polarization (Arras \& Lai 1999, 
and references therein).Magnetic dipole radiation from an off-centered dipole (Harrison \& Tademaru 1975) requires very rapid initial rotation $(P \sim 1 \mathrm{~ms})$, but an asymmetric matter-loaded jet from a rotating neutron star should be a more effective source of linear momentum (DT92). Such a jet could be driven by transient accretion through a disk.

There are fewer theoretical models for neutron star rotation and they are less well developed. Burrows \& Hayes (1996) speculated that large-scale convective plumes might be responsible for generating both rotation and kicks; Phinney \& Spruit (1998) recently suggested that off-center neutrino kicks imparted at the contracting $v$-sphere might play a similar role. An argument against models in which pulsar rotation is driven by torques acting at small radii comes from the inference that the initial rotational kinetic energy $E_{\mathrm{rot}} \sim 10^{50}\left(P_{\mathrm{ns}} / 10 \mathrm{~ms}\right)^{-2}$ ergs must exceed the translational kinetic energy $10^{48}\left(\mathrm{~V} / 300 \mathrm{~km} \mathrm{~s}^{-1}\right)^{2}$ ergs by 1 to 2 orders of magnitude in several radio pulsars. This large rotational kinetic energy is naturally acquired from torques acting near the outer boundary of the collapsing core. It is difficult to understand how off-center neutrino kicks could result in a much larger rotational kinetic energy, as suggested recently by Hansen (1999).

The relative merits of these scenarios deserve some comment. Hydrodynamical stresses alone appear insufficient to induce the required dipole asymmetry in the neutrino flux. The convection zone inside the forming neutron star is surrounded by an extended, stably stratified layer. The convective Mach number does not exceed $M_{\text {con }} \sim 0.1$ (KJM), which implies a fractional anisotropy $M_{\text {con }}^{2} \sim 10^{-2}$ in the neutrino flux. Symmetry breaking is more easily achieved via hydromagnetic stresses. A direct scaling to rapidly rotating M-dwarfs (which have deep convective zones and develop large, long-lived polar magnetic spots: Vogt 1988) suggests that neutron stars with initial spin periods of a few milliseconds will develop polar magnetic spots that coherently modulate the neutrino flux over many rotation periods (DT92). Suppose that the dipole magnetic flux is concentrated in asymmetric polar spots of solid angle $\Delta \Omega_{\text {spot }}$ and flux density $B^{2} / 4 \pi \sim B_{\text {sat }}^{2} / 4 \pi \equiv \rho V_{\text {con }}^{2}$ (which correspond to $\left.B_{\text {sat }} \sim 10^{16}\left(\rho / \rho_{\text {nuc }}\right)^{1 / 2} V_{\text {con8 }} \quad G\right)$. The linear momentum acquired by the neutron star after it has radiated energy $E_{v} \sim 3 \times 10^{53}$ ergs in neutrinos is

$$
M_{\mathrm{ns}} V_{\mathrm{ns}} \sim \frac{E_{v}}{c}\left(\frac{\tau_{\mathrm{spot}}}{\tau_{\mathrm{KH}}}\right)^{1 / 2}\left(\frac{\Delta \Omega_{\mathrm{spot}}}{4 \pi}\right) .
$$

Here $\tau_{\text {spot }} / \tau_{\mathrm{KH}}$ is the coherence time of the spot in units of the Kelvin timescale of the neutron star. The dipole field ${ }^{8}$ corresponding to a proper motion $V_{\mathrm{ns}}$ is

$$
B_{\text {dipole }}>B_{\text {sat }}\left(\frac{\Delta \Omega_{\text {spot }}}{2 \pi}\right)=2\left(\frac{M_{\mathrm{ns}} V_{\mathrm{ns}} c}{E_{v}}\right)\left(\frac{\tau_{\mathrm{spot}}}{\tau_{\mathrm{KH}}}\right)^{-1 / 2} B_{\mathrm{sat}},
$$

or, equivalently,

$$
\begin{aligned}
B_{\text {dipole }}> & 5 \times 10^{14}\left(\frac{V_{\mathrm{ns}}}{1000 \mathrm{~km} \mathrm{~s}^{-1}}\right) V_{\text {con } 8} \\
& \times\left(\frac{\rho}{\rho_{\text {nuc }}}\right)^{1 / 2}\left(\frac{\tau_{\text {spot }}}{\tau_{\mathrm{KH}}}\right)^{-1 / 2} \mathrm{G} .
\end{aligned}
$$

\footnotetext{
${ }^{8}$ Averaged over the surface of the neutron star.
}

The dipole field remaining in the star after the convective motions turn off could be lower than this bound. In comparison, kick models involving asymmetric neutrino emission driven by weak parity violation require a much stronger dipole field $\left(\sim 10^{16} \mathrm{G}\right.$ according to the latest calculation by Arras \& Lai 1999).

All these mechanisms for generating rotation and kicks are based on some intrinsic property of the supernova core, such as vigorous convection, or a high neutrino flux; but each is tied only indirectly to the supernova mechanism itself. Because rapid rotation and large proper motions appear to be fairly generic properties of young neutron stars, it would be more satisfying if the supernova mechanism made this connection more directly. With this goal in mind, we now consider the response of the outer part of the collapsing core to an asymmetric Rayleigh-Taylor expansion of the accretion shock.

A spherical shell of low-density fluid, sandwiched between the central neutron core and the accretion shock, represents a valid solution to the equation of hydrostatic equilibrium. This solution is, however, unstable to an asymmetric, sideways displacement of the shell. The equilibrium shape of the resulting Rayleigh-Taylor plume, enveloped by a quasi-static accretion shock, was calculated in $\S 4.3$. This calculation ceases to be valid after the plume reaches the radius $R_{\text {exp }}$ (eq. [67]) and heating is too rapid for the shock to remain quasi-static. Outside this radius, which lies slightly interior to the position $R_{R}$ of the rarefaction wave (eq. [57]), the shock begins to run. The net result is an off-center explosion.

According to Newton's third law, material initially at rest with respect to the neutron core imparts no net momentum after falling in ballistically. The exchange of momentum between an expanding aspherical shock and the central core thus depends, crucially, on the initial velocity of the infalling material with respect to the core. It will be assumed that the pre-supernova core is initially spherical, so that the velocity relative to the neutron core vanishes before the shock reaches the rarefaction wave. Burrows \& Hayes (1996) have considered a more extreme situation where the supernova shock propagates through a core that is, ab initio, missing material in one direction. They obtain kick velocities approaching $1000 \mathrm{~km} \mathrm{~s}^{-1}$.

The kick is dominated by the last material to be captured by the core before the shock assumes an almost spherical shape. ${ }^{9}$ The initial infall velocity relative to the core can be estimated by assuming that the collapse is delayed for a time comparable to the infall time of the unperturbed core at radius $R_{\exp }$, which is about twice the free-fall time (Yahil \& Lattimer 1982),

$$
\tau_{\text {delay }} \simeq \frac{\pi}{2^{1 / 2}}\left(\frac{R_{\text {exp }}^{3}}{G M_{\text {core }}}\right)^{1 / 2} .
$$

Integrating the gravitational pull of this uncollapsed material over the hemisphere $0 \leq \theta \leq \pi / 2$ opposite to the plume gives the net kick

$$
V_{\mathrm{ns}}=\tau_{\text {delay }} \int_{R_{\text {exp }}}^{\infty} R^{2} d R \int_{0}^{\pi / 2} 2 \pi \sin \theta d \theta\left[\frac{G \rho_{0}(R)}{R^{2}} \cos \theta\right]
$$

\footnotetext{
${ }^{9}$ Late infall triggered by the partial reflection of the shock off density discontinuities in the outer core (e.g., Chevalier 1989) contributes little to the kick because the specific gravitational binding energy is small where the shock reflects.
} 
away from the plume. The integral works out to

$$
\begin{aligned}
V_{\mathrm{ns}}= & \frac{\pi^{2}}{2^{3 / 2}}\left(\frac{\mathscr{M}}{M_{\text {core }}}\right)\left(\frac{G M_{\text {core }}}{R_{\text {exp }}}\right)^{1 / 2} \\
= & 360\left(\frac{R_{\exp }}{2 \times 10^{3} \mathrm{~km}}\right)^{-1 / 2}\left(\frac{\mathscr{M}_{31}}{3}\right) \\
& \times\left(\frac{M_{\text {core }}}{1.4 M_{\odot}}\right)^{-1 / 2} \mathrm{~km} \mathrm{~s}^{-1} .
\end{aligned}
$$

Note the relatively weak dependence on $R_{\text {exp }}$. A kick velocity approaching $1000 \mathrm{~km} \mathrm{~s}^{-1}$ requires a dense core, $\mathscr{M}>10^{32} \mathrm{~g} \mathrm{~cm}^{-3}$. This suggests that in some magnetars with very large proper motions, such as the soft gamma repeaters $1900+14$ and $0526-66$, an additional physical mechanism (such as neutrino starspots; eq. [92]) contributes to the kick.

The existence of a net circulation,

$$
\Gamma \equiv \oint \boldsymbol{V} \cdot \boldsymbol{d l}
$$

about the neutron core requires that the expanding Rayleigh-Taylor plume depart from axial symmetry. To this end, consider the motion of the Rayleigh-Taylor spikes of core material near the outer boundary of the plume. These spikes descend through the lower density M-fluid at nearly the free-fall speed when the Atwood number $\left(\rho-\rho_{M}\right) /$ $\left(\rho+\rho_{M}\right)$ is close to unity (Alon et al. 1994). The pressure stratification in the plume gives a characteristic angular spacing $\Delta \Omega_{\text {spike }} \sim\left(\ell_{P} / R\right)^{2} \sim 1 / 16$. (The spacing is even larger, $\Delta \Omega_{\text {spike }} \sim 1$, in two-dimensional simulations of neutrino-driven convection; JM96.) The spikes are deflected from the radial direction by an angle $\delta \theta$, and their specific angular momentum is $\left(2 G M_{\text {core }} R_{\text {exp }}\right)^{1 / 2} \delta \theta$. Assuming the angular momenta of individual spikes to be randomly oriented, the mean specific angular momentum of the accretion flow is

$$
\begin{aligned}
\frac{\Gamma}{2 \pi} & \equiv \varepsilon_{\text {rot }}\left(G M_{\text {core }} R_{\text {exp }}\right)^{1 / 2} \\
& \sim\left(2 G M_{\text {core }} R_{\text {exp }}\right)^{1 / 2} \delta \theta\left(\frac{\Delta \Omega_{\text {spike }}}{\Delta \Omega_{M}}\right)^{1 / 2} .
\end{aligned}
$$

The dimensionless parameter $\varepsilon_{\text {rot }}$ then takes the value

$$
\varepsilon_{\mathrm{rot}} \sim 0.06\left(\frac{\delta \theta}{0.1}\right)\left(\frac{\Delta \Omega_{M}}{2 \pi}\right)^{-1 / 2} .
$$

The infalling material is guaranteed to form a quasiKeplerian disk in orbit around the neutron star if

$$
\varepsilon_{\text {rot }}>\sqrt{\frac{R_{v}}{R_{\exp }}} \sim 8 \times 10^{-2} .
$$

This lower bound is conservative: if the angular momentum is distributed inhomogeneously through the collapsing material, then a disk forms at lower average rotation rates. This is the case if the infalling material is heated sufficiently to become hydrostatically supported and undergoes a bar instability before its outer portions collapse to the surface of the newly formed neutron star. We consider the interaction of such a disk with the rapidly rotating neutron star in the next section.
What deserves to be emphasized here, despite the uncertainty in this estimate, is that the net circulation in equation (98) exceeds the value needed to generate the spins of young, fast pulsars. Given a total infalling mass $\sim 4 \pi \mathscr{M}$,

$$
\varepsilon_{\text {rot }}\left(G M_{\text {core }} R_{\text {exp }}\right)^{1 / 2} 4 \pi \mathscr{M} \simeq I_{\text {ns }} \frac{2 \pi}{P_{\text {ns }}},
$$

one sees that an initial spin period $P_{\mathrm{ns}} \sim 20 \mathrm{~ms}$ corresponds to

$$
\begin{aligned}
\varepsilon_{\mathrm{rot}}= & 0.05 I_{\mathrm{ns} 45}\left(\frac{P_{\mathrm{ns}}}{20 \mathrm{~ms}}\right)^{-1}\left(\frac{R_{\exp 8}}{2}\right)^{-1 / 2} \\
& \times\left(\frac{\mathscr{M}_{31}}{3}\right)^{-1}\left(\frac{M_{\mathrm{ns}}}{1.4 M_{\odot}}\right)^{-1 / 2} .
\end{aligned}
$$

When $\varepsilon_{\text {rot }}$ exceeds the value in equation (99), the spin angular momentum can be directly related (eq. [95]) to the mass $\Delta M_{\text {rot }}$ of centrifugally supported material (Chevalier 1989). While fallback continues, and for some time thereafter, the accretion flow through the disk maintains a strong enough ram to reach the surface of the star-even in the extreme case where the dipole magnetic field is amplified by internal convective motions to $\sim 10^{15} \mathrm{G}$. The specific angular momentum of the accreted material is $\left(G M_{\mathrm{ns}} R_{\mathrm{ns}}\right)^{1 / 2}$, and the increase in the spin angular momentum of the star is

$$
I_{\mathrm{ns}} \Delta \Omega=\Delta M_{\mathrm{rot}} \sqrt{G M_{\mathrm{ns}} R_{\mathrm{ns}}} .
$$

The corresponding spin period is

$$
P_{\mathrm{ns}} \leq 0.023 I_{\mathrm{ns} 45} R_{\mathrm{ns} 6}^{-1 / 2}\left(\frac{\Delta M_{\mathrm{rot}}}{10^{-2} M_{\odot}}\right)^{-1}\left(\frac{M_{\mathrm{ns}}}{1.4 M_{\odot}}\right)^{-1 / 2} \mathrm{~s} .
$$

\section{FURTHER OBSERVATIONAL CONSEQUENCES}

\subsection{Implications for Shock Nucleosynthesis}

Observations of the synthesis of ${ }^{56} \mathrm{Ni}$ and other irongroup elements provide important clues to the development of the supernova shock. The total amount of ${ }^{56} \mathrm{Ni}$ synthesized (as measured through its decay to ${ }^{56} \mathrm{Co}$ and ${ }^{56} \mathrm{Fe}$ ) constrains the position of the cut between ejected and collapsed mass (WW95; Thielemann et al. 1996). One deduces $M\left({ }^{56} \mathrm{Ni}\right) \sim 0.075 M_{\odot}$ for SN 1987a and several other Type II/Ib SNe (Arnett et al. 1989; Young, Baron, \& Branch 1995; Clocchiatti \& Wheeler 1997), with much lower masses inferred for a few others (e.g., $M\left({ }^{56} \mathrm{Ni}\right) \sim 0.002 M_{\odot}$ for $\mathrm{SN}$ 1997D; Turatto et al. 1998). The amount of ${ }^{56} \mathrm{Ni}$ synthesized relative to ${ }^{54} \mathrm{Fe},{ }^{57} \mathrm{Co}$, and ${ }^{58} \mathrm{Ni}$ is a sensitive probe of the neutron excess $\Delta Y_{n}=Y_{n}-0.5$. The abundances of these isotopes (and their decay products) suggest that $\Delta Y_{n} \lesssim$ $5 \times 10^{-3}$ in the parts of core-collapse supernovae undergoing explosive nucleosynthesis. In this regard, the accumulation of low-density M-fluid below the shock ( $\$ \$ 4.2,4.3$, 4.4) has two beneficial effects: it reduces the ejected mass of neutronized material (for a fixed energy); and it reduces the electron degeneracy in the ejected material, thus raising the rate of $e^{+}$captures relative to $e^{-}$captures.

The largest contribution to $\Delta Y_{n}$ comes from buoyant material that rises upward from the gain region (near the radius $R_{\text {shock }}$ of the original stalled shock). At this small a radius, $\alpha$-particles are entirely dissociated (eq. [9]), the electrons in the shocked material are nondegenerate, and the 
equilibrium neutron excess is determined by a balance between $\bar{v}_{e}$ absorption on protons and $v_{e}$ absorption on neutrons, $\left(\frac{1}{2}-\Delta Y_{n}\right) L_{\bar{v}_{e}}\left\langle\varepsilon_{\bar{v}_{e}}\right\rangle=\left(\frac{1}{2}+\Delta Y_{n}\right) L_{v_{e}}\left\langle\varepsilon_{v_{e}}\right\rangle$. This yields (e.g., Bethe 1997)

$$
\Delta Y_{n}=\frac{1}{2}\left[\frac{L_{v_{e}}\left\langle\varepsilon_{v_{e}}\right\rangle-L_{\bar{v}_{e}}\left\langle\varepsilon_{\bar{v}_{e}}\right\rangle}{L_{v_{e}}\left\langle\varepsilon_{v_{e}}\right\rangle+L_{\bar{v}_{e}}\left\langle\varepsilon_{\bar{v}_{e}}\right\rangle}\right] \simeq 0.025 .
$$

The M-fluid has an even higher entropy and lower neutron excess than the surrounding fluid. The pressure in radiation (and pairs) is related to the accretion ram by $P_{e^{ \pm}}+P_{\gamma}=$ $11 / 4\left(a T_{2}^{4}\right) \simeq(1+\beta)^{-1}\left[\rho_{1} V_{1}^{2}\right]\left(R_{\text {sphere }}\right), \quad$ where $\quad \beta=$ $B^{2} / 8 \pi\left(P_{e^{ \pm}}+P_{\gamma}\right)$ and the postshock temperature $T_{2}$ is given by equation (9). The entropy in radiation and pairs is then

$$
\begin{aligned}
S_{\text {rad }}= & 4 \frac{\left(P_{e^{ \pm}}+P_{\gamma}\right)_{2}}{\rho_{M} T_{2} / m_{n}}=\frac{8}{1+\beta} \frac{G M_{\text {core }} m_{n}}{R_{\text {sphere }} T_{2}}\left(\frac{\rho_{1}}{\rho_{M}}\right)_{R_{\text {sphere }}} \\
= & 11(1+\beta)^{-3 / 4}\left(\frac{5 \rho_{1}}{\rho_{M}}\right)_{R_{\text {sphere }}}\left(\frac{\dot{M}}{M_{\odot} \mathrm{s}^{-1}}\right)^{-1 / 4} \\
& \times\left(\frac{R_{\text {sphere }}}{100 \mathrm{~km}}\right)^{-3 / 8}\left(\frac{M_{\text {core }}}{1.4 M_{\odot}}\right)^{7 / 8}
\end{aligned}
$$

per baryon. By contrast, simulations of successful neutrinodriven shocks show that the convective material has much larger $\Delta Y_{n}$ because it is advected upward from layers where the electrons are mildly degenerate.

An estimate of the mass $M(\mathrm{n}-$ rich $)$ of (slightly) neutronrich material injected into the Rayleigh-Taylor plume is obtained by relating $M\left(\mathrm{n}-\right.$ rich) to the energy $E_{0}$ ejected into the plume. Because the expanding plume does work on the accretion flow, and only a fraction $\delta_{M}$ of this work is returned to it ( $\$ 4.2), E_{0}$ is larger by a factor $1+2 \delta_{M}$ than the energy $E$ received by the shock. The energy per baryon in relativistic particles and magnetic field at the base of the plume is

$$
\frac{3+\beta}{1+\beta}\left(\frac{\rho_{1} V_{1}^{2}}{\rho_{M}}\right)_{R_{\text {sphere }}},
$$

so the ejected mass is

$$
\begin{aligned}
M(\mathrm{n}-\mathrm{rich})= & \frac{(1+\beta)\left(1+2 \delta_{M}\right)}{3+\beta} \frac{E}{V_{1}^{2}\left(R_{\text {sphere }}\right)}\left(\frac{\rho_{M}}{\rho_{1}}\right)_{R_{\text {sphere }}} \\
= & 1.4 \times 10^{-2} E_{51} \frac{(1+\beta)\left(1+2 \delta_{M}\right)}{3+\beta}\left(\frac{R_{\text {sphere }}}{100 \mathrm{~km}}\right) \\
& \times\left(\frac{\rho_{M}}{\rho_{1}}\right)_{R_{\text {sphere }}}\left(\frac{M_{\text {core }}}{1.4 M_{\odot}}\right)^{-1} M_{\odot} .
\end{aligned}
$$

When this slightly neutron-rich material is ejected and mixed with core material composed of $\alpha$-rich nuclei $\left(\Delta Y_{n}=\right.$ 0 ), the average neutron excess is further reduced. One finds

$$
\begin{aligned}
\Delta Y_{n} & =\Delta Y_{n}(\text { ejecta }) \frac{M(\text { ejecta })}{M(\text { ejecta })+M(\alpha-\text { rich })} \\
& \simeq 1 \times 10^{-3}
\end{aligned}
$$

for $M($ ejecta $) \sim 1 \times 10^{-2} M_{\odot}$ and $M(\alpha-$ rich $) \sim 0.2 M_{\odot}$.

The ejected mass of ${ }^{56} \mathrm{Ni}$ is reduced by the reverse shocks that form where the supernova shock crosses the $\mathrm{Si} / \mathrm{O}$ and $\mathrm{He} / \mathrm{H}$ interfaces (WW95; Janka \& Müller 1995). Without this effect, $M\left({ }^{56} \mathrm{Ni}\right)$ may be a few times too large. The amount of material undergoing complete $\mathrm{Si}$ burning $\left(T>4 \times 10^{9} \mathrm{~K}\right)$ is directly related to the mass parameter $\mathscr{M}$ in the density profile of the outer collapsing core and is, in order of magnitude, $M\left({ }^{56} \mathrm{Ni}\right) \sim 4 \pi \mathscr{M}=0.2\left(\mathscr{M}_{31} / 3\right) M_{\odot}$. If the shock is powered by asymmetric accretion, it continues to gain energy after reaching the position of the rarefaction wave. As a result, it will have less energy than $\sim 10^{51}$ ergs when the temperature drops to $T=4 \times 10^{9} \mathrm{~K}$. However, the enclosed mass grows slowly with radius in this part of the collapsing core, and the mass of synthesized ${ }^{56} \mathrm{Ni}$ is probably not much reduced below the calculations for fast, neutrino-heated shocks.

Finally it should be noted that the mass of ejected, slightly neutron-rich material is far in excess of the estimated mass of $\sim 10^{-5} M_{\odot}$ in $r$-process material per supernova (Woosley \& Hoffman 1992). Its entropy is probably too small to maintain a large neutron excess (eq. [105]) as the temperature falls below $\sim 1 \mathrm{MeV}$.

\subsection{Remnant Magnetic Fields in the Supernova Ejecta}

These considerations also strongly limit direct ejection of hot, magnetized material from the supernova core. As the shock expands through the stellar core, it decelerates where the mass per logarithm of radius $\left[4 \pi \rho(R) R^{3}\right]$ increases with radius. These zones are situated outside the helium/ hydrogen interface and next to compositional discontinuities in the core (WW95). When the shock decelerates, magnetized fluid falls behind and is buried by the freshly shocked stellar material. Only strongly collimated, relativistic material will be able to punch through the stellar envelope (see MacFadyen \& Woosley 1999; Khokhlov et al. 1999 ), and only then if the progenitor is relatively compact.

The magnetic field interior to the expanding shock will, nonetheless, be advected outward. It is potentially observable in the supernova remnant. If the shock succeeds, when the magnetic energy accumulated inside the rarefaction wave (radius $R_{R}$; eq. [57]) reaches a critical value, then one can directly relate the remnant field to the ejecta energy ${ }^{10} E$ :

$$
\frac{B^{2}\left(R_{R}\right)}{8 \pi}\left(\frac{4 \pi}{3}\right) R_{R}^{3}=E .
$$

As the shock decelerates inside the supernova core, and a reverse shock forms inside it, the M-fluid may become trapped inside the reverse shock. Nonetheless, the successful ejection of $r$-process material during the last stages of neutrino heating provides direct evidence that buoyancy forces allow some of this material (which has a higher mass and a lower entropy than the $r$-process material; $\S 6.1$ ) to rise past the reverse shock and be ejected.

Conservation of magnetic flux implies a nebular field

$$
B_{\exp }(t)=B\left(R_{R}\right)\left(\frac{R_{R}}{V_{\mathrm{ej}} t}\right)^{2},
$$

where $V_{\mathrm{ej}}$ is the asymptotic speed of the inner edge of the unbound material. Combining equations (57), (109), and (110), we obtain

$$
\begin{aligned}
B_{\text {exp }}(t)= & 2 \times 10^{-3} E_{51}^{1 / 2}\left(\frac{t}{1 \mathrm{yr}}\right)^{-2}\left(\frac{V_{\mathrm{ej}}}{10^{4} \mathrm{~km} \mathrm{~s}^{-1}}\right)^{-2} \\
& \times\left[\frac{M\left(<R_{R}\right)}{1.6 M_{\odot}}\right]^{1 / 6}\left(\frac{t_{\mathrm{exp}}}{1 \mathrm{~s}}\right)^{1 / 3} \mathrm{G} .
\end{aligned}
$$

\footnotetext{
follows.
} 
Here $t_{\exp }$ is the time at which the shock passes the rarefaction wave.

It is also useful to compare this passively advected magnetic field with the field injected into the supernova remnant by a rapidly rotating pulsar (the spindown luminosity of which is assumed to remain constant at early times). This energy accumulates at a rate

$$
\frac{d E_{B}}{d t}=-\frac{E_{B}}{t}+L_{\mathrm{psr}}
$$

which yields $E_{B}=\frac{1}{2} L_{\mathrm{psr}} t$. A direct comparison with the above expression shows that the pulsar-generated field

$$
B_{\mathrm{psr}}=\frac{\left(6 E_{B}\right)^{1 / 2}}{\left(V_{\mathrm{ej}} t\right)^{3 / 2}}
$$

dominates on a timescale $t>10 \mathrm{yr}$, if $L_{\mathrm{psr}} \sim L_{\mathrm{Crab}}=$ $5 \times 10^{38}$ ergs s$^{-1}$ :

$$
\begin{aligned}
\frac{B_{\mathrm{psr}}}{B_{\mathrm{exp}}}= & 20 E_{51}^{-1 / 2}\left(\frac{L_{\mathrm{psr}}}{L_{\mathrm{Crab}}}\right)^{1 / 2}\left(\frac{t}{1 \mathrm{yr}}\right)\left(\frac{V_{\mathrm{ej}}}{10^{4} \mathrm{~km} \mathrm{~s}^{-1}}\right)^{1 / 2} \\
& \times\left[\frac{M\left(<R_{R}\right)}{1.6 M_{\odot}}\right]^{-1 / 6}\left(\frac{t_{\mathrm{exp}}}{1 \mathrm{~s}}\right)^{-1 / 3}
\end{aligned}
$$

The author thanks Norm Murray, Chris Fryer, Ewald Müller, and Jim Wilson for conversations and the Sloan Foundation for financial support. The referee kindly provided comments that helped focus the presentation of the paper.

\section{APPENDIX}

Our main goal in this appendix is to address the stability of a spherical accretion shock that surrounds a volume of low-density and high-entropy fluid (labeled $M$ ). We calculate the dispersion relations from equations (73), (74) and (75) for long-wavelength perturbations of the shock (and the thin shell of dense, shocked fluid sitting just below it).

We first consider the thin-shell regime, where the mass $M_{\text {sh }}$ of the thin shell of shocked fluid is much less than in equation (68). We focus on the contribution of the acceleration of the shock $\partial^{2}\left(\delta R_{S}\right) / \partial t^{2}$ to the pressure perturbation $\delta P_{M}$ just below the thin shell of shocked fluid (eq. [70]). The unperturbed equation of hydrostatic equilibrium is

$$
\frac{1}{\rho_{M}} \frac{\partial P_{M}}{\partial R}=-\frac{G M_{\text {core }}}{R^{2}} .
$$

The perturbed continuity and Euler equations in the M-fluid are

$$
\frac{\partial \delta \rho_{M}}{\partial t}+\rho_{M} \nabla \cdot V_{M}+V_{M, R} \frac{\partial \rho_{M}}{\partial R}=0
$$

and

$$
\frac{\partial V_{M}}{\partial t}=-\frac{1}{\rho_{M}} \nabla \delta P_{M}-\frac{\delta \rho_{M}}{\rho_{M}} \frac{G M_{\text {core }}}{R^{2}} \hat{\boldsymbol{R}}
$$

Taking

$$
\delta P_{M}(R, \theta, \phi)=\delta \widetilde{P}_{M}(R) Y_{\ell m}(\theta, \phi)
$$

and

$$
\delta \rho_{M}=\frac{\rho_{M}}{\Gamma P_{M}} \delta P_{M}
$$

these equations can be combined to yield

$$
\omega^{2}\left(\frac{\rho_{M}}{\Gamma P_{M}}\right) \delta \tilde{P}_{M}+\frac{1}{R^{2}} \frac{\partial}{\partial R}\left(R^{2} \frac{\partial \delta \tilde{P}_{M}}{\partial R}\right)-\frac{\ell(\ell+1)}{R^{2}} \delta \tilde{P}_{M}+\frac{\partial}{\partial R}\left(\frac{\rho_{M}}{\Gamma P_{M}} \delta \widetilde{P}_{M}\right) \frac{G M_{\text {core }}}{R^{2}}=0 .
$$

Making use of the approximate solution to equation (A1),

$$
\frac{P_{M}}{\rho_{M}}=\frac{\Gamma-1}{\Gamma} \frac{G M_{\text {core }}}{R},
$$

this equation simplifies to

$$
\frac{\omega^{2}}{(\Gamma-1) G M_{\text {core }} / R^{3}} \delta \tilde{P}_{M}+R^{2} \frac{\partial^{2} \delta \tilde{P}_{M}}{\partial R^{2}}+\frac{1}{\Gamma-1} R \frac{\partial \delta \tilde{P}_{M}}{\partial R}+\left[\frac{1}{\Gamma-1}-\ell(\ell+1)\right] \delta \widetilde{P}_{M}=0 .
$$

Near marginal stability, we can substitute $\omega=0$ in equation (A8) and find the power-law solution

$$
\delta \tilde{P}_{M}(R)=\delta \tilde{P}_{M}\left(R_{\mathrm{sh}}\right)\left(\frac{R}{R_{\mathrm{sh}}}\right)^{\alpha},
$$


where

$$
\alpha=-\frac{\Gamma}{2(\Gamma-1)}+\frac{1}{2} \sqrt{\frac{\Gamma^{2}-4 \Gamma+4}{(\Gamma-1)^{2}}+4 \ell(\ell+1)} .
$$

For $\Gamma=4 / 3$ and $\ell=1$, this is

$$
\alpha=-2+\sqrt{3}
$$

whereas for large $\ell$,

$$
\alpha \simeq \ell-\frac{\Gamma}{2(\Gamma-1)} .
$$

The solution to the perturbed Euler equation (71) is now

$$
\delta P_{M}\left(R_{\mathrm{sh}}\right)=\omega^{2} \frac{\rho_{M}\left(R_{\mathrm{sh}}\right) R_{\mathrm{sh}}^{2}}{\mathscr{R} /(2 \Gamma)+\alpha},
$$

where

$$
\mathscr{R} \equiv \frac{\rho_{M}\left(R_{\mathrm{sh}}\right)}{\rho_{1}\left(R_{\mathrm{sh}}\right)} .
$$

The pressure balance at the shock (eqs. [69] and [70]) becomes

$$
\frac{\omega^{2}}{G M_{\text {core }} / R_{\text {sh }}^{3}}=\left(\mathscr{R}-5-i 2^{3 / 2} \frac{\omega}{\left(G M_{\text {core }} / R_{\text {sh }}^{3}\right)^{1 / 2}}\right)\left(\frac{1}{2 \Gamma}+\frac{\alpha}{\mathscr{R}}\right) \text {. }
$$

This equation yields equations (73) and (74).

Now let us turn to the thick-shell regime- $M_{\mathrm{sh}}$ much greater than in equation (68)-where the inertia limiting the displacement of the shock is dominated by the shell of shocked fluid. The equations governing the surface density $\sigma$ and nonradial velocity $V_{T}$ of the shell are easily modified from those presented in Vishniac (1983) to include the additional accretion velocity $V_{1}$ just outside the shock,

$$
\begin{gathered}
\frac{\partial \sigma}{\partial t}=-\left(\frac{2}{R_{\mathrm{sh}}} \frac{\partial R_{\mathrm{sh}}}{\partial t}\right) \sigma+\rho_{1}\left[\frac{\partial R_{\mathrm{sh}}}{\partial t}+V_{1}\left(R_{\mathrm{sh}}\right)\right]-\left(\nabla_{T} \cdot V_{T}\right) \sigma \\
\sigma \frac{\partial^{2} R_{\mathrm{sh}}}{\partial t^{2}}=P_{M}\left(R_{\mathrm{sh}}\right)-\rho_{1}\left[\frac{\partial R_{\mathrm{sh}}}{\partial t}+V_{1}\left(R_{\mathrm{sh}}\right)\right]^{2}-\sigma \frac{G M_{\mathrm{core}}}{R_{\mathrm{sh}}^{2}}
\end{gathered}
$$

and

$$
\frac{\partial \boldsymbol{V}_{T}}{\partial t}=-\frac{\rho_{1}}{\sigma}\left[\frac{\partial R_{\mathrm{sh}}}{\partial t}+V_{1}\left(R_{\mathrm{sh}}\right)\right] \boldsymbol{V}_{T}-\left(\frac{1}{R_{\mathrm{sh}}} \frac{\partial R_{\mathrm{sh}}}{\partial t}\right) \boldsymbol{V}_{T}-\frac{c_{s}^{2}}{\sigma} \nabla_{T} \sigma-\frac{P_{M}\left(R_{\mathrm{sh}}\right)}{\sigma} \nabla_{T} R_{\mathrm{sh}} .
$$

In the thick-shell regime, $\sigma \gg \rho_{1} R_{\mathrm{sh}}$, and these equations simplify. The second to last term on the right-hand side of equation (A18) is smaller than the last term by a factor $\sim 1 / \mathscr{R}$ and can also be ignored. We then obtain the following simple equations for the perturbed variables $\delta=\sigma / \sigma_{0}-1, \delta R_{\mathrm{sh}}$, and $V_{T}$ (where $\sigma_{0}$ is the unperturbed surface density):

$$
\begin{gathered}
\frac{\partial \delta}{\partial t}=\frac{1}{R_{\mathrm{sh}}} \frac{\partial \delta R_{\mathrm{sh}}}{\partial t}-\nabla_{T} \cdot V_{T} ; \\
\frac{\partial^{2} \delta R_{\mathrm{sh}}}{\partial t^{2}}=-\left(\frac{G M_{\text {core }}}{R_{\mathrm{sh}}^{3}}\right) \delta ;
\end{gathered}
$$

and

$$
\frac{\partial V_{T}}{\partial t}=-\frac{P_{M}\left(R_{M}\right)}{\sigma_{0}} \nabla_{T}\left(\delta R_{\mathrm{sh}}\right) .
$$

The equation of hydrostatic equilibrium implies that the pressure just interior to the thick shell is

$$
P_{M}\left(R_{M}\right) \simeq \sigma_{0} \frac{G M_{\text {core }}}{R_{\text {sh }}^{2}}
$$

Taking all quantities to vary with time as $e^{-i \omega t}$, these equations can be combined to yield

$$
\omega^{4}-\omega^{2} \frac{G M_{\text {core }}}{R_{\mathrm{sh}}^{3}}=\ell(\ell+1)\left(\frac{G M_{\text {core }}}{R_{\mathrm{sh}}^{3}}\right)^{2},
$$

with the solution in equation (75). 


\section{REFERENCES}

Alon, U., Hecht, J., Ofer, D., \& Shvarts, D. 1994, Phys. Rev. Lett., 74, 534

Arnett, W. D., Bahcall, J. N., Kirshner, R. P., \& Woosley, S. E. 1989, ARA\&A, 27, 629

Arras, P. \& Lai, D. 1999, ApJ, 519, 745

Baade, W., \& Zwicky, F. 1934, Proc. Natl. Acad. Sci., 20, 254

Batchelor, G. K. 1970, Fluid Dynamics (Cambridge: Cambridge Univ. Press)

Bethe, H. A. 1990, Rev. Mod. Phys., 62, 801

.1997, ApJ, 490, 765

Bethe, H. A., \& Wilson, J. R. 1985, ApJ, 295, 14

Brown, G. E. 1995, ApJ, 440, 270

Brown, G. E., \& Bethe, H. A. 1994, ApJ, 423, 659

Bruenn, S. W. 1993, in Nuclear Physics in the Universe, ed. M. W. Guidry \& M. R. Strayer (Bristol: IOP), 31

Burrows, A. 1987, ApJ, 318, L57

Burrows, A., Hayes, J., \& Fryxell, B. A. 1995, ApJ, 450, 830 (BHF)

Burrows, A., \& Hayes, J. 1996, Phys. Rev. Lett., 76, 352

Chevalier, R. A. 1989, ApJ, 346, 847

Chevalier, R. A., Blondin, J. M., \& Emmering, R. T. 1992, ApJ, 392, 118

Chevalier, R. A., \& Klein, R. I. 1978, ApJ, 219, 994

Clocchiatti, A., \& Wheeler, J. C. 1997, ApJ, 491, 375

Colgate, S. A., \& Johnson, M. H. 1960, Phys. Rev. Lett., 5, 235

Colgate, S. A., \& White, R. H. 1966, ApJ, 143, 626

Cordes, J. M., \& Chernoff, D. F. 1998, ApJ, 505, 315

De Nisco, K. R., Bruenn, S. W., \& Mezzacappa, A. 1998, in Stellar Evolution, Stellar Explosions, and Galactic Chemical Evolution, ed. A. Mezzacappa (Bristol: IOP), 571

Duncan, R. C., \& Thompson, C. 1992, ApJ, 392, L9 (DT92)

Durney, B. R., de Young, D. S., \& Roxburgh, I. A. 1993, Sol. Phys., 145, 207

Epstein, R. I. 1979, MNRAS, 188, 305

Fahlman, G. G., \& Gregory, P. C. 1981, Nature, 293, 202

Frail, D. A. 1998, in The Many Faces of Neutron Stars, ed. R. Buccheri, J. van Paradijs, \& M. A. Alpar (Boston: Dordrecht), 179

Frail, D. A., \& Kulkarni, S. R. 1991, Nature, 352, 785

Fryer, C., Burrows, A., \& Benz, W. 1998, ApJ, 496, 333

Goldreich, P., Murray, N., Willette G., \& Kumar, P. 1991, ApJ, 370, 752

Goldreich, P., \& Weber, S. V. 1980, ApJ, 238, 991

Gotthelf, E. V., \& Vasisht, G. 1997, ApJ, 486, L133

Goodman, J. 1990, ApJ, 358, 214

Hansen, B. 1999, ApJ, 512, L117

Hansen, B., \& Phinney, E. S. 1997, MNRAS, 291, 569

Harrison, E. R., \& Tademaru, E. 1975, ApJ, 201, 447

Herant, M., Benz, W., \& Colgate, S. 1992, ApJ, 395, 642

Houck, J. C., \& Chevalier, R. A. 1991, ApJ, 376, 234

Hurley, K., Kouveliotou, C., Cline, T., Mazets, E., Golenetskii, S., Frederiks, D. D., \& Van Paradijs, J. 1999, ApJ, 523, L37

Janka, H. T., \& Monchmeyer, R. 1989, A\&A, 226, 69

Janka, H. T., \& Müller, E. 1994, A\&A, 290, 496

Janka, H. T., \& Müller, E. 1995, ApJ, 448, L109
Janka, H. T., \& Müller, E. 1996, A\&A, 306, 167 (JM96)

Jun, B.-I. 1998, ApJ, 499, 282

Jun, B.-I., Norman, M. L., \& Stone, J. M. 1995, ApJ, 453, 332

Keil, W., Janka, H.-T., \& Müller, E. 1996, ApJ, 473, L111 (KJM)

Khokhlov, A. M., Hoeflich, P. A., Oran, E. S., Wheeler, J. C., \& Wang, L. 1999, ApJ, 524, L107

Kouveliotou, C., et al. 1998, Nature, 393, 235

Kouveliotou, C., et al. 1999, ApJ, 510, L115

Kulkarni, S. R. 1992, Philos. Trans. R. Soc. London, A, 341, 77

Kulkarni, S. R., Frail, D., Kassim, N., Murakami, T., \& Vasisht, G. 1994, Nature, 368,129

Leblanc, J. M., \& Wilson, J. R. 1970, ApJ, 161, 541

Lyne, A. G., \& Lorimer, D. R. 1994, Nature, 369, 127

MacFadyen, A., \& Woosley, S. E. 1999, ApJ, 524, 262

Mazurek, T. J., Cooperstein, J., \& Kahana S. 1982, in Supernovae: A Survey of Current Research, ed. M. J. Rees \& R. J. Stoneham (Dordrecht: Reidel), 71

Meneguzzi, M., \& Pouquet, A. 1989, J. Fluid Mech., 205, 297

Mezzacappa, A., Calder, A. C., Bruenn, S. W., Blondin, J. M., Guidry,

M. W., Strayer, M. R., \& Ulmar, A. S. 1998, ApJ, 493, 848 (M98)

Miller, D. S., Wilson, J. R., \& Mayle, R. W. 1993, ApJ, 415, 278

Murray, N. 1992, Sol. Phys., 138, 419

Nakamura, T. 1998, Prog. Theor. Phys., 100, 921

Phinney, E. S., \& Spruit, H. C. 1998, Nature, 393, 139

Ruzmaikin, A. A., \& Sokoloff, D. D. 1981, Soviet Astron. Lett., 7, 388

Schinder, P. J., Schramm, D. N., Wiita, P. J., Margolis, S. H., \& Tubbs, D. L. 1987, ApJ, 313, 531

Thielemann, F.-K., Nomoto, K., \& Hashimoto, M. 1996, ApJ, 460, 408

Thompson, C. 1994, MNRAS, 270, 480

Thompson, C., \& Duncan, R. C. 1993, ApJ, 408, 194 (TD93)

Thompson, C., \& Blaes, O. 1998, Phys. Rev. D, 57, 3219

Thompson, C., Duncan, R. C., Woods, P. M., Kouveliotou, C., Finger, M. H., \& van Paradijs, J. 1999, preprint (astro-ph/9908086)

Turatto, M., et al. 1998, ApJ, 498, L129

Van Kerkwijk, M. H., Kulkarni, S. R., Matthews, K., \& Neugebauer G. 1995, ApJ, 444, L33

Vasisht, G., Kulkarni, S. R., Frail, D. A., \& Greiner, J. 1994, ApJ, 431, L35

Vishniac, E. T. 1983, ApJ, 274, 152

Vogt, S. S. 1988, in The Impact of Very High S/N Spectroscopy on Stellar Physics, ed. G. Cayrel de Strobel \& M. Spite (Dordrecht: Kluwer), 253

Wilson, J. R., \& Mayle, R. W. 1993, Phys. Rep., 227, 97

Woosley, S. E. 1987, in The Origin and Evolution of Neutron Stars, ed. D. J. Helfand \& J.-H. Huang (Dordrecht: Reidel), 255

Woosley, S. E., \& Hoffman, R. D. 1992, ApJ, 395, 202

Woosley, S. E., \& Weaver, T. A. 1995, ApJS, 101, 181 (WW95)

Yahil, A., \& Lattimer, J. M. 1982, in Supernovae: A Survey of Current Research, ed. M. J. Rees \& R. J. Stoneham (Dordrecht: Reidel), 53

Young, T. R., Baron, E., \& Branch, D. 1995, ApJ, 449, L51 